\title{
Synthesis of daunosamyl anthraquinone and anthracyclinone analogs
}

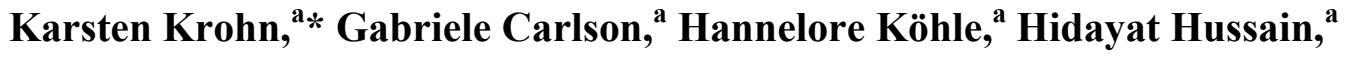 \\ and Ivan R. Green ${ }^{b}$ \\ ${ }^{a}$ Department of Chemistry, University of Paderborn, Warburger Straße 100, 33098 Paderborn, \\ Germany \\ ${ }^{b}$ Department of Chemistry, University of the Western Cape, P/Bag x17, Bellville, 7530, South \\ Africa \\ E-mail: k.krohn@uni-paderborn.de
}

\begin{abstract}
Four groups of aglycones (cyclopenta-annulated anthraquinones, mono- and bis-hydroxyalkylanthraquinones, steffinycinone) were glycosylated with daunosamine chloride. In all cases, the major products were the respective $\alpha$-glycosides $(\alpha, \beta$-ratio ca. 9:1). In some cases, the $\beta$ glycosides rearranged to furanosides under the glycosylation conditions.
\end{abstract}

Keywords: Glycosylation, non-natural anthracyclines, daunosamine, rearrangement

\section{Introduction}

New synthetic anthracyclines continue to be of great interest to overcome resistance and to reduce toxicity of these important anti-cancer drugs. ${ }^{1}$ In connection with our work on the total synthesis of anthracyclinones (for reviews, see refs 2-13) and the aglycones of the important anthracycline antitumor antibiotics (for reviews, see refs 14-36), we started an investigation on the glycosylation of structurally diverse synthetic anthraquinone derivatives prepared by our group. (For a selection of other syntheses of anthracycline derivatives see refs 37-47). The general aim of this research was to get insight into the DNA binding capability as a function of distinct structural parameters and to correlate these binding data with cytotoxic activity. This investigation will be published elsewhere. In the present study we, describe the glycosylation step, the ratio of the $\alpha$ - to $\beta$-glycosides formed, and a new rearrangement of the $N$-trifluoroacetyl daunosamine $\beta$-glycosides to the respective furanoside glycosides. Trifluoroacetyl-protected daunosamine chloride $\mathbf{2}$ was used as the sugar component in all the reactions (Scheme 1). The activated protected sugar $\mathbf{2}$ was prepared from daunosamine hydrochloride, a gift from Prof. Arcamone, and was also prepared on a larger scale using the procedure of Horton and 
Weckerle. ${ }^{48}$ Silver triflate, introduced by Hanessian into glycosylation chemistry, ${ }^{49}$ served as the catalyst, as described by Arcamone et al. in the early stages of synthetic anthracycline analogs. ${ }^{50 a}$ Daunosamine was selected as the sugar component because previous work showed clearly that this sugar provided the essential stereochemical requirements for DNA binding and biological activity. $^{50-52}$

\section{Results and Discussion}

The aglycones selected can be classified into four groups: (a) a tetracyclic cyclopentaanthraquinone (Scheme 1), (b) mono-hydroxymethyl anthraquinones (Scheme 2), (c) bishydroxymethyl anthraquinones (Scheme 4), and (d) steffimycynone, an anthraquinone with an unusual substitution in ring D (Scheme 5).

The first aglycone, the cyclopenta-anthraquinone, rac-1, was prepared stereoselectively by reaction of leuco-quinizarine with $\alpha, \beta$-unsaturated aldehydes such as methacrolein (see the Experimental Section). The trans-configuration of the methyl and hydroxyl group was deduced from the coupling constants of $J_{1,2}=3.4 \mathrm{~Hz}$ in the ${ }^{1} \mathrm{H}$ NMR spectrum. From the glycosylation of rac-1, only a single $\alpha$-glycoside, 3 , was isolated in pure form and the assignment shown in Scheme 1 is tentative.<smiles>C[C@H]1Cc2c(O)c3c(c(O)c2[C@@H]1O)C(=O)c1ccccc1C3=O</smiles>

rac-1

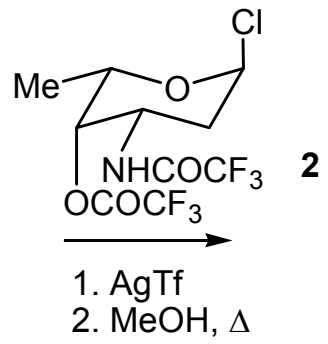

2. $\mathrm{MeOH}, \Delta$

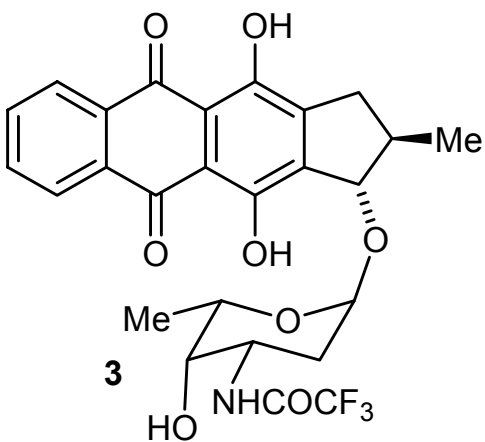

Scheme 1. Glycosylation of cylopenta-anthraquinone rac-1.

The largest group of non-natural daunosamyl glycosides was prepared from achiral hydroxymethyl anthraquinones 4-10 and the chiral racemic 1-hydroxybutylanthraquinone, rac27. The aglycones were readily prepared by a variation of the Marschalk reaction (reaction of dihydroanthraquinones with aldehydes) in which reductive elimination of the benzylic hydroxyl group was prevented by conducting the hydroxyalkylation of the dihydroanthraquinones at low temperature, with shorter reaction times, and with rapid re-oxidation of the dihydroquinones to the respective anthraquinones. ${ }^{53-56}$ The glycosylation reactions were studied in great detail in repeated reactions and by careful analysis of the reaction products. In the reaction of the enantiomerically pure halo-sugar 2 with the achiral hydroxymethyl anthraquinones 4-10, only the 
$\alpha$-glycosides and the respective $\beta$-glycosides were expected. In fact, in many cases these two products (11-17: $\alpha$-glycosides) and (18-22: $\beta$-glycosides) could be isolated, with the $\alpha$-glycosides being mostly the less polar products (Scheme 2). However, careful analysis of some of the polar reaction products revealed a surprising phenomenon. The compounds 23-26 turned out to be furanosides as shown in Scheme 2. In two cases (aglycone 7 and 9), no $\beta$-glycoside could be isolated from the reaction mixture. In contrast, all three possible products were isolated from the reaction of aglycones 8 and 10. In the case of the chiral 1-hydroxybutyl-quinizarine rac-27, the glycoslyation led to the diastereoisomeric $\alpha$-glycosides $\mathbf{2 8}$ and 29. The minor $\beta$-glycoside were not isolated in this case, and the stereochemical assignment is arbitrary.

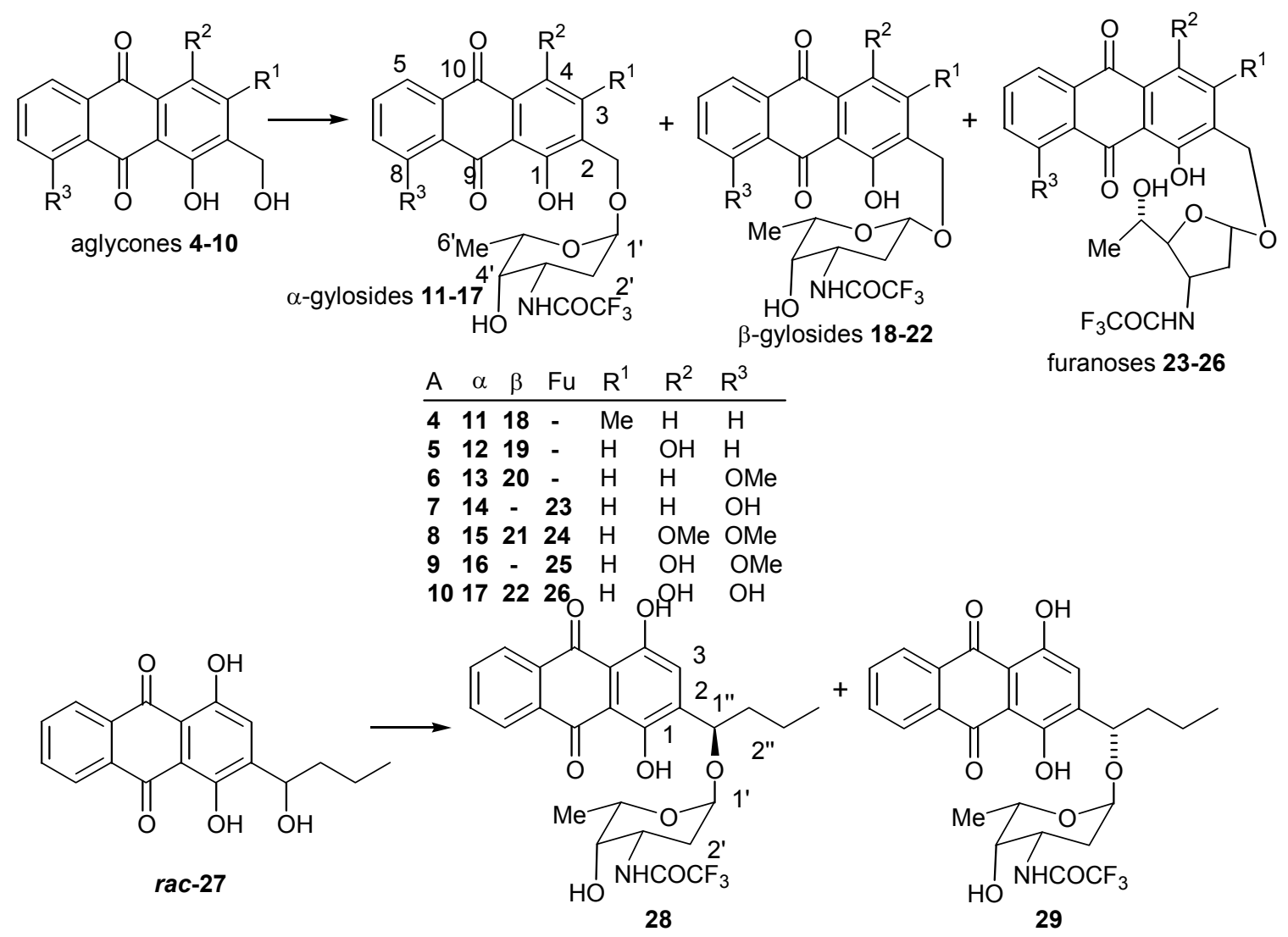

Scheme 2. Glycosylation of achiral-(4-10) and racemic-(rac-27) hydroxyalkyl-anthraquinones.

The configuration of the furanosides 23-26 could in part be solved by comparison with the stereoisomeric methyl 2,3,6-trideoxy-3-(trifluoroacetamido)- $\beta$ - and - $\alpha$-D-ribo-hexo-furanosides, $\mathbf{A}$ and $\mathbf{B}$, and the $\beta$ - and $\alpha$-L-lyxo-furanosides $\mathbf{C}$ and $\mathbf{D}$ (Scheme 3), prepared by El Khadem and Matsuura via the corresponding dithioacetals of epidaunosamin and daunosamine, respectively. ${ }^{57}$ As can be seen from Table 1, in which the relevant ${ }^{1} \mathrm{H}$ NMR data of the furanoses A-D was obtained from the literature ${ }^{57}$ are compared with those of our furanoses 23-26, the coupling constant, $J=4.0 \mathrm{~Hz}$, for the $\beta$-glycoside A compares well with that of our furanose glycosides $(J$ 
$=4.0-4.2)$. In addition, the corresponding signals for the anomeric protons of the $\alpha$-glycosides $\mathbf{B}$ and $\mathbf{D}$ appear as multiplets with two different coupling constants (see Table 1). Thus, the $\beta$ configuration of our furanosides can be deduced unambiguously. On the assumption that the other stereocenters of the original daunosamine sugar did not change, the $\beta$-L lyxo configurations for 23-26 and 34 are proposed as shown in Schemes 2 and 4. The data shown in Table 1 also show the consistency of the relevant ${ }^{1} \mathrm{H}$ NMR data of the synthetic furanoses, demonstrating their stereochemical identity.

The mechanism of the rearrangement of the hexoses into the furanoses during the silverpromoted glycosylation is not yet clear. However, since the major $\alpha$-pyranosides are stable under the reaction conditions, a rearrangement of the $\beta$-anomers during the glycosylation reaction, or the presence of a furanoside structure of the daunosamyl halide, seems most probable.

Table 1. ${ }^{1} \mathrm{H}-\mathrm{NMR}$ data for assignment of the configuration of the furanoses 23-26 and $\mathbf{3 4}$

\begin{tabular}{lclllllll}
\hline Cpd & Config. & 1'-H; m, $J$ & 2 'a-H & 2 'e-H & 3'-H & 4 '-H & 5 '-H & 6 '-H \\
\hline $\mathbf{2 3}$ & $\beta$ & $5.44 ; \mathrm{d}, 4.2$ & 2.05 & 2.32 & 4.55 & 3.88 & 3.84 & 1.28 \\
$\mathbf{2 4}$ & $\beta$ & $5.44 ; \mathrm{d}, 4.2$ & 2.05 & 2.32 & 4.55 & 3.88 & 3.84 & 1.28 \\
$\mathbf{2 5}$ & $\beta$ & $5.44 ; \mathrm{d}, 4.2$ & 2.05 & 2.32 & 4.55 & 3.88 & 3.84 & 1.30 \\
$\mathbf{2 6}$ & $\beta$ & $5.45 ; \mathrm{d}, 4.0$ & 2.06 & 2.33 & 4.58 & 3.89 & 3.85 & 1.29 \\
$\mathbf{3 4}$ & $\beta$ & $5.44 ; \mathrm{d}, 4.0$ & 2.08 & 2.28 & 4.53 & 3.86 & 3.83 & 1.27 \\
$\mathbf{A}^{\mathbf{a}}$ & $\beta$ & $5.19 ; \mathrm{d}, 4.0$ & $1.79-2.54$ & $1.79-2.54$ & 4.64 & $3.66-4.11$ & 3.40 & 1.23 \\
$\mathbf{B}^{\mathbf{a}}$ & $\alpha$ & $5.20 ; \mathrm{m}, 1.5,5.4$ & $1.87-2.81$ & $1.87-2.81$ & 4.75 & $3.70-4.15$ & 3.43 & 1.26 \\
$\mathbf{C}^{\mathbf{a}}$ & $\beta$ & $5.20 ;-$ & $1.76-2.52$ & $1.76-2.52$ & 4.52 & $3.69-4.01$ & 3.46 & 1.27 \\
$\mathbf{D}^{\mathbf{a}}$ & $\alpha$ & $5.13 ; \mathrm{m}, 2.3,4.7$ & $1.76-2.33$ & $1.76-2.33$ & 4.65 & $3.64-4.01$ & 3.45 & 1.19 \\
\hline
\end{tabular}

${ }^{\mathrm{a}}$ From reference 57 .

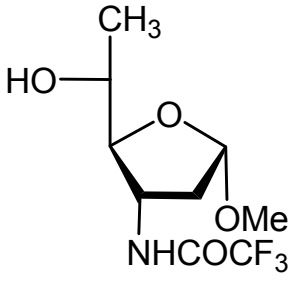

A

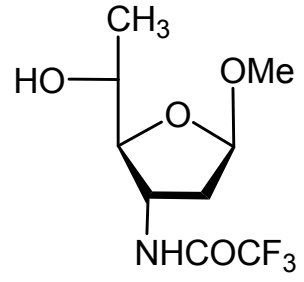

B

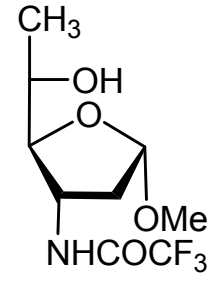

C

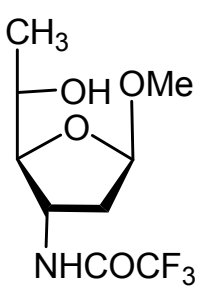

D

Scheme 3. Structures of furanoses A-D prepared from daunosamine and epidaunosamime. ${ }^{57}$

Two examples of bis-hydroxymethylated achiral anthraquinones $\mathbf{3 0}$ and $\mathbf{3 2}$ were reacted with an excess of the sugar halide 2 . The bis-glycosides are very attractive candidates to probe the possibility of stronger intercalation into double-stranded DNA. Since in principle each glycoside can appear in three different forms, a complex mixture was expected. However, to our delight, in the case of the 2,3-bis-hydroxymethyl-quinizarine $\mathbf{3 0},{ }^{55}$ which has both hydroxymethyl groups 
on the same side of the molecule, one major bis- $\alpha$-glycoside $\mathbf{3 1}$ was isolated in highly pure form. However, in the case of bis-hydroxymethyl-1,5-dihydroxy-anthraquinone $\mathbf{3 2},{ }^{54}$ where the two hydroxymethyl groups are on opposite sides and on different aryl rings of the molecular scaffold, careful analysis of the glycosylation products revealed the formation of two major products $\mathbf{3 3}$ and 34. In the analogue 33, both sugar moieties were attached in $\alpha$-glycosidic form, whereas in the analogue 34 , one $\alpha$-glycoside and one rearranged $\beta$ - L-lyxo-furanoside were present in the same molecule.

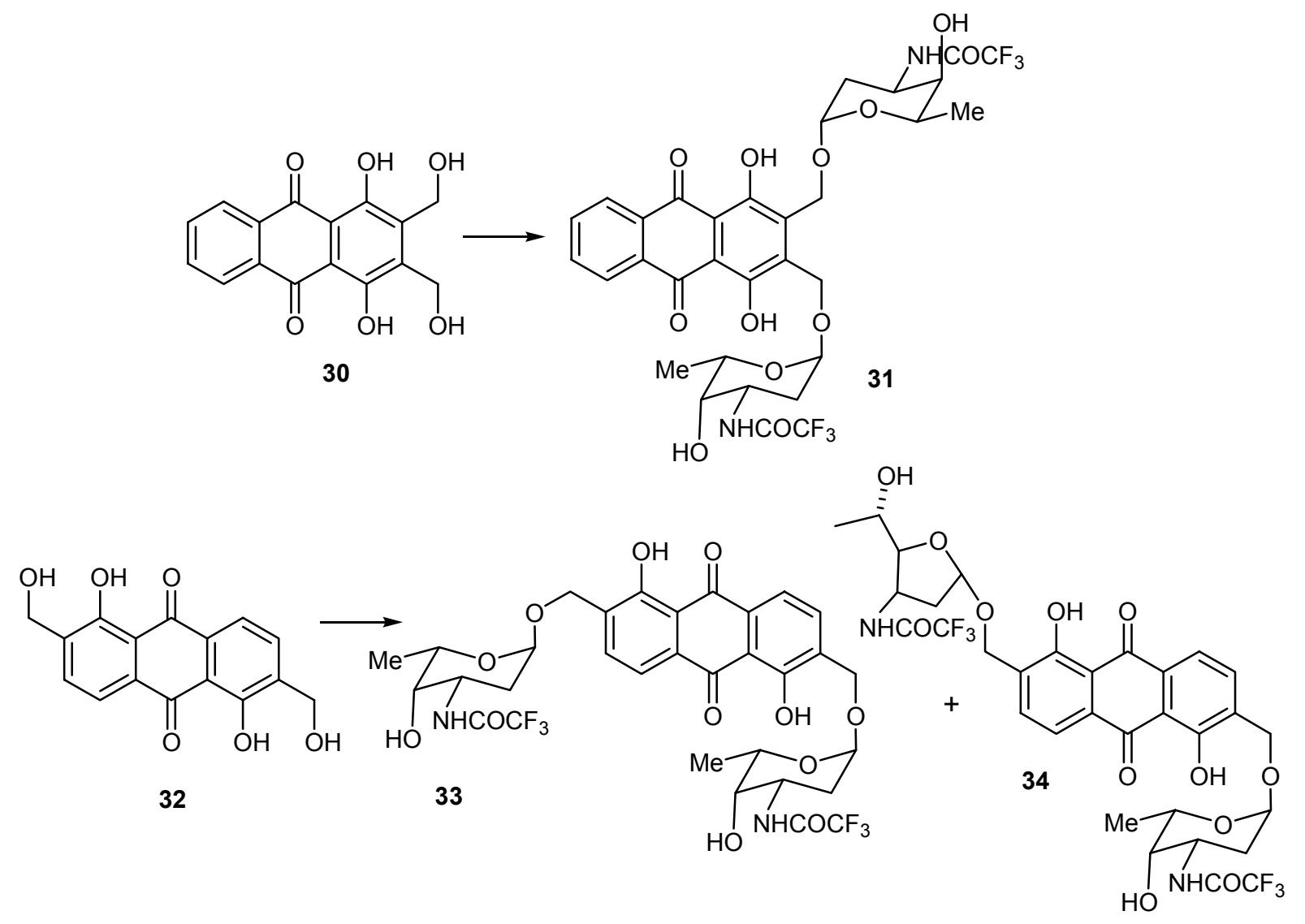

Scheme 4. Glycosylation of bis-hydroxymethylated anthraquinones $\mathbf{3 0}$ and $\mathbf{3 2}$.

Finally, the naturally occurring anthracyclinone steffimycinone, $35,{ }^{58}$ was converted into the daunosamyl glycoside 36 by reaction with the halo-sugar 2 (Scheme 5). As expected, the homochiral aglycone was converted into one major $\alpha$-glycoside, as seen from the ${ }^{1} \mathrm{H}$ NMR spectrum. Since the natural anthracycline steffimycin does not have an amino sugar moiety, for reasons of comparison, it was considered advantageous to have the homologous series of daunosamyl glycosides. In addition, the hybrid glycoside 34 offered the chance to probe the influence of the different substitution pattern of the hydroaromatic ring A, quite different from the standard daunorubicine, on DNA binding, and also to compare the amino-sugar glycoside 36 with the natural steffimycins ${ }^{58}$ which have no amino-sugar moiety. 
<smiles>COc1cc(O)c2c(c1)C(=O)c1cc3c(c(O)c1C2=O)[C@@H](O)[C@H](OC)[C@@](C)(O)C3=O</smiles>

steffimycinone (35)

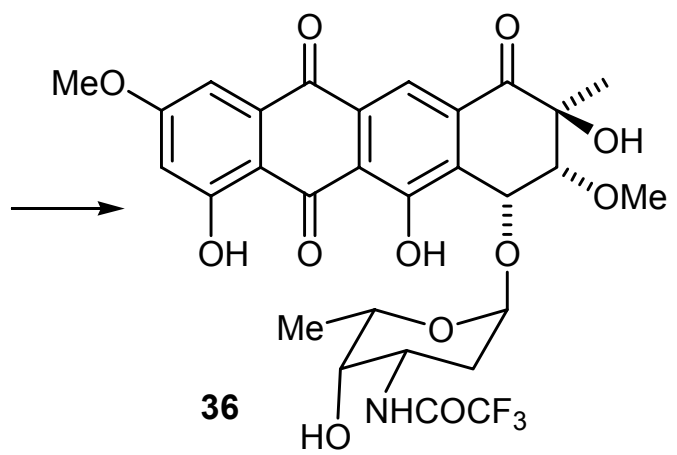

Scheme 5. Conversion of steffimycinone $\mathbf{3 5}$, into the daunosamyl $\alpha$-glycoside $\mathbf{3 6}$.

In summary, the four groups of selected aglycones were converted into daunosamyl glycosides. In all cases, the major products were the $\alpha$-glycosides ( $\alpha: \beta$-ratio ca 3-9:1). In five cases, the $\beta$-glycosides rearranged to $\beta$-L-lyxo-furanosides under the glycosylation conditions employed.

\section{Experimental Section}

General Procedures. Melting points were determined with an Electrothermal apparatus and are uncorrected. The IR spectra $(\mathrm{KBr})$ were measured with a Perkin-Elmer 297, and the NMR spectra $\left(\mathrm{CDCl}_{3}\right)$ with a Bruker WH $270(270 \mathrm{MHz})$ or Bruker Avance-500 NMR spectrometer, with TMS as internal standard ( $m c=$ centered multiplet). EI mass spectra were obtained on a MAT 8200 mass spectrometer at $70 \mathrm{eV}$. UV spectra were measured with a Perkin-Elmer Lambda 45 instrument and the UV/VIS data of representative compounds are listed in Table 2.

General procedure for glycoside synthesis. ${ }^{51} \mathrm{~A}$ solution (or suspension) of the respective aglycone in dry $\mathrm{CH}_{2} \mathrm{Cl}_{2}$ (distilled over $\left.\mathrm{P}_{2} \mathrm{O}_{5}\right)(10-30 \mathrm{~mL}$ ) was treated first with a threefold molar excess of daunosamyl chloride (2), prepared according to the procedure of Horton and Weckerle. ${ }^{48}$ Then a solution of silver trifluorosulfonate (threefold excess) in dry diethyl ether (2$5 \mathrm{~mL}$ ) was added dropwise. The mixture was stirred under exclusion of light and monitored by TLC (for reaction times see the individual compounds). The reaction was quenched by addition of excess aq. $\mathrm{NaHCO}_{3}$ solution $(\mathrm{ca} .5 \mathrm{~mL})$ and the organic phase was dried $\left(\mathrm{Na}_{2} \mathrm{SO}_{4}\right)$ and evaporated at reduced pressure. To cleave the trifluoroacetate ester, the residue was dissolved in dry methanol $(20 \mathrm{~mL})$ and heated under reflux for $30 \mathrm{~min}$. The solvent was removed under reduced pressure, the residue redissolved in $\mathrm{CH}_{2} \mathrm{Cl}_{2}(10 \mathrm{~mL})$, washed twice with water $(10 \mathrm{~mL})$, dried $\left(\mathrm{Na}_{2} \mathrm{SO}_{4}\right)$ and separated by preparative TLC $(1 \mathrm{~mm}$ silica gel plates, Schleicher and Schüll). $\mathrm{CH}_{2} \mathrm{Cl}_{2}$ was normally used as the eluent; for more polar compounds $15-25 \%$ of diethyl ether-, and for very polar compounds an additional 1-2 \% of methanol, was added. The plates 
were developed 15-20 times to separate the very similar isomeric glycosides. The major products usually consisted of the diastereomeric $\alpha$-L-glycosides, and the minor components were either the $\beta$-L-glycosides or rearranged $\beta$-furanoside. Some of the glycosides could be crystallized from diethyl ether while others were precipitated with petroleum to afford a noncrystalline powder. These powders showed a melting range of $120-130{ }^{\circ} \mathrm{C}$. All samples were dried for $6-7 \mathrm{~h}$ at $60{ }^{\circ} \mathrm{C}$ under high vacuum $(1 \mathrm{~mm} \mathrm{Hg})$. In some cases, traces of solvent $\left(\mathrm{CH}_{2} \mathrm{Cl}_{2}\right.$, diethyl ether) could not be removed completely without decomposition of the glycosides.

Table 2. UV/VIS data of representative chromophores

\begin{tabular}{cl}
\hline Compound & \multicolumn{1}{c}{$\lambda_{\max } / \mathrm{nm}\left(\log _{10} \varepsilon\right)$} \\
\hline $\mathbf{3}$ & $516 \mathrm{sh}(3.75), 481(3.98), 331 \mathrm{sh}(3.41), 289(3.97), 252(4.16), 218(3.15)$ \\
$\mathbf{1 1}$ & $409(2.83), 329(3.51), 284 \mathrm{sh}(4.09), 262(4,17), 218(4.15)$ \\
$\mathbf{1 2}$ & $478(4.05), 380 \mathrm{sh}(3.47), 284(4.00), 245(4.19), 218(4.19)$ \\
$\mathbf{1 3}$ & $415(4.01), 254(4.24), 232(420), 217(4.23)$ \\
$\mathbf{1 4}$ & $433(4.05), 286(4.10), 250(4.19), 218(4.18)$ \\
$\mathbf{1 6}$ & $476(4.05), 380(3.48), 284(3.99), 248(4.21), 237(4.21), 218(4.21)$ \\
$\mathbf{3 4}$ & $438(4.10), 424(4.10), 290(3.06), 256(4.33), 233(4.31), 218(4.32)$ \\
\hline
\end{tabular}

1,4,11-Trihydroxy-2-methylcyclopenta[b]anthracene-5,10-dione (rac-1). A solution of leucoquinizarine (9,10-dihydroquinizarine) $(3.60 \mathrm{~g}, 15 \mathrm{mmol})$ in $1 \mathrm{~N} \mathrm{NaOH}(200 \mathrm{~mL})$ had added a solution of methacrolein $(1 \mathrm{~mL})$ in methanol $(20 \mathrm{~mL})$, dropwise, under nitrogen at $0{ }^{\circ} \mathrm{C}$. After stirring for $2 \mathrm{~h}$ at $0{ }^{\circ} \mathrm{C}$, the solution was acidified by addition of $2 \mathrm{~N} \mathrm{HCl}(110 \mathrm{~mL})$ and the precipitate was filtered off. The dried crude product was filtered through a short column of silica gel (15 x $3 \mathrm{~cm}$; dichloromethane). The eluate was concentrated at reduced pressure to $10 \mathrm{~mL}$ and diluted with petroleum ether until a precipitate formed. After $14 \mathrm{~h}$ at $4{ }^{\circ} \mathrm{C}, 3.35 \mathrm{~g}(72 \%)$ of the cyclopenta[b]anthraquinone rac-1 crystallized; mp 230-231 ${ }^{\circ} \mathrm{C}$. IR: $3500(\mathrm{OH}), 1613$ (quinone, chelated), $1583 \mathrm{~cm}^{-1}$ (aromatic). ${ }^{1} \mathrm{H} \mathrm{NMR}(270 \mathrm{MHz}): \delta=1.32\left(\mathrm{~d}, J=7.0 \mathrm{~Hz}, 3 \mathrm{H}, \mathrm{CH}_{3}\right), 2.40$ $2.76(\mathrm{~m}, 2 \mathrm{H}, 2-\mathrm{H}, 3-\mathrm{H}), 2.93(\mathrm{~s}, 1 \mathrm{H}, \mathrm{OH}), 3.33(\mathrm{~m}, 1 \mathrm{H}, 3-\mathrm{H}), 5.11(\mathrm{~d}, J=3.8 \mathrm{~Hz}, 1 \mathrm{H}, 1-\mathrm{H}), 7.83$ $(\mathrm{m}, 2 \mathrm{H}, 7-\mathrm{H}, 8-\mathrm{H}), 8.33(\mathrm{~m}, 2 \mathrm{H}, 6-\mathrm{H}, 9-\mathrm{H}), 13.03(\mathrm{~s}, 1 \mathrm{H}, \mathrm{OH}), 13.18$ (s, 1H, OH). UV (methanol): $\lambda_{\max }(\log \mathrm{e})=250$ (4.56), 2.88 (3.91), 449 (3.85), 4.78 (3.92), $512 \mathrm{~nm}$ (3.69). MS $\left(160{ }^{\circ} \mathrm{C}\right): m / e=311(25 \%, \mathrm{M}+1), 310$ (97, M+), 294 (14), 293(35), 292 (100), 291 (36), 281 (11), 276 (35), 274 (14), 263 (13), 262 (10), 249 (12), 187 (11), 165 (13). Anal. Calcd for $\mathrm{C}_{18} \mathrm{H}_{14} \mathrm{O}_{5}$ (310.31): C, 69.67; H, 4.55. Found: C, 69.77; H, 4.52\%.

\section{1-O-(2,3,6-Trideoxy-3-trifluoroacetamido- $\alpha-L-l y x o-h e x o p y r a n o s y l)-4,11-d i h y d r o x y-2-$} methylcyclopenta[b]anthracene-5,10-dione (3). The aglycone $\mathbf{r a c - 1}(82 \mathrm{mg}, 0.2 \mathrm{mmol}$ ) was reacted as described in the General Procedure to afford $36 \mathrm{mg}(34 \%)$ of glycoside 3; mp $302{ }^{\circ} \mathrm{C}$. IR: $3420(\mathrm{OH}, \mathrm{NH}), 1720,1697(\mathrm{C}=\mathrm{O}), 1620$ (quinone, chelated), $1589 \mathrm{~cm}^{-1}$ (Ar). ${ }^{1} \mathrm{H}$ NMR (500 $\left.\mathrm{MHz}, \mathrm{CDCl}_{3}\right): \delta 1.21\left(\mathrm{~d}, J=7.1 \mathrm{~Hz}, 3 \mathrm{H}, 2-\mathrm{CH}_{3}\right), 1.31\left(\mathrm{~d}, J=6.6 \mathrm{~Hz}, 3 \mathrm{H}, 6{ }^{\prime}-\mathrm{H}\right), 1.82(\mathrm{dt}, J=$ 16.0, 13.0, 3.5 Hz, 1H, 2'b-H), 2.00 (m, 2H, 2'a-H), 2.54 (dd, $J=17.5,4.6 \mathrm{~Hz}, 1 \mathrm{H}, 3 \mathrm{~b}-\mathrm{H}), 2.60$ 
(m, 1H, 2-H), 3.38 (ddd, $J=17.5,7.5,0.7 \mathrm{~Hz}, 1 \mathrm{H}, 3 \mathrm{a}-\mathrm{H}), 3.64$ (br d, $J=7.6,6.5 \mathrm{~Hz}, 1 \mathrm{H}, 4^{\prime}-\mathrm{H}$ ), 4.23 (br q, $\left.J=6.5 \mathrm{~Hz}, 1 \mathrm{H}, 5^{\prime}-\mathrm{H}\right), 4.40$ (m, 1H, 3'-H), 4.95 (d, $\left.J=3.4 \mathrm{~Hz}, 1 \mathrm{H}, 1-\mathrm{H}\right), 5.48$ (d, $J=$ $3.5 \mathrm{~Hz}, 1 \mathrm{H}, 1$ '-H), 6.66 (br d, $J=8.7 \mathrm{~Hz}, 1 \mathrm{H}, \mathrm{NH}), 7.80$ (m, 2H, 7-H, 8-H), 8.35 (m, 6-H, 9-H), $13.00(\mathrm{~s}, 1 \mathrm{H}, \mathrm{OH}), 13.39(\mathrm{~s}, 1 \mathrm{H}, \mathrm{OH}) ;{ }^{13} \mathrm{C} \mathrm{NMR}\left(125 \mathrm{MHz}, \mathrm{CDCl}_{3}\right): \delta=16.6$ (q, C-6'), 18.9 (q, $\mathrm{CH}_{2} \mathrm{CH}_{3}$ ), 29.6 (t, C-2'), 36.0 (t, C-3), 40.6 (d, C-2), 46.2 (d, C-3'), 65.9 (d, C-5'), 69.2 (d, C-4'), 86.5 (d, C-1), 97.4 (d, C-1'), 112.6 (s, C-11a), 113.2 (s, C-5a), 126.9 (s, C-5, C-8), 133.5 (s, C11a), 133.6 (s, C-9a), 134.3 (d, C-8), 134.4 (d, C-7), 141.5 (s, C-13), 145.1 (s, C-4), 155.0 (s, C12), 156.0 (s, C-5), 186.9 (s, C-11), 187.2 (s, C-10); EIMS $\left(290{ }^{\circ} \mathrm{C}\right): m / z(\%)=535(11)[\mathrm{M}+]$, 310 (100); 292 (94), 187 (36). HREIMS: $m / z(\%)=535.1456$ (Calcd. for, 535.1454). Anal. Calcd for $\mathrm{C}_{26} \mathrm{H}_{24} \mathrm{NO}_{8} \mathrm{~F}_{3}$ (535.48): C, 58.32; H, 4.52; N, 2.62. Found: C, 58.07; H, 4.53; N, $2.59 \%$.

\section{1-Hydroxy-3-methyl-2-[1-O-(2',3',6'-trideoxy-3'-trifluoroacetamido- $\alpha$-L-lyxo-hexo-}

pyranosyl)-methyl]-9,10-anthraquinone (11). Aglycone $4^{54,60}(200 \mathrm{mg}, 0.75 \mathrm{mmol})$ was reacted according to the general procedure with daunosamyl chloride (2) (573 $\mathrm{mg}, 1.32 \mathrm{mmol}$ ) and silver triflate $(410 \mathrm{mg}, 1.59 \mathrm{mmol})$, to afford starting material $(11 \mathrm{mg}, 0.04 \mathrm{mmol})$ and the less polar $\alpha$-glycoside 11 (282.8 mg, 81.4\%), mp 238-239 ${ }^{\circ} \mathrm{C}$. IR (KBr): 3450, $3300(\mathrm{OH}, \mathrm{NH})$, 2980, $2920(\mathrm{CH}), 1705(\mathrm{C}=\mathrm{O}), 1685$ (quinone), 1649 (quinone, chelated), 1595, 1560, 1357, $1185,1025,712 \mathrm{~cm}^{-1} .\left(500 \mathrm{MHz}, \mathrm{CDCl}_{3}\right): \delta=1.25$ (d, $\left.J=6.5 \mathrm{~Hz}, 3 \mathrm{H}, 6{ }^{\prime}-\mathrm{H}\right), 1.81$ (ddd, $J=12.5$, 10.1, 3.5 Hz, 1H, 2'-a-H), 1.98 (dd, $\left.J=12.5 \mathrm{~Hz}, 4.5 \mathrm{~Hz}, 1 \mathrm{H}, 2^{\prime} \mathrm{e}-\mathrm{H}\right), 2.55$ (s, 3H, 11-H), 3.59 (br d, $\left.J=5 \mathrm{~Hz}, 1 \mathrm{H}, 4^{\prime}-\mathrm{H}\right), 4.20$ (q, $\left.J=6.5 \mathrm{~Hz}, 1 \mathrm{H}, 5 '-\mathrm{H}\right), 4.39$ (m, 1H, 3'-H), 4.65 (d, $J=11.0 \mathrm{~Hz}$, $\left.1 \mathrm{H}, \mathrm{CH}_{2}\right), 4.95$ (d, J=11.0 Hz, 1H, $\left.\mathrm{CH}_{2}\right), 5.07$ (d, $\left.J=3.5 \mathrm{~Hz}, 1 \mathrm{H}, \mathrm{l}^{\prime}-\mathrm{H}\right), 6.69$ (d, $J=8.3 \mathrm{~Hz}, 1 \mathrm{H}$, 4'-NH), 7.69 (s, 1H, 4-H), 7.82 (m, 2H, 6-H, 7-H), 8.32 (m, 2H, 5-H, 8-H), 13.12 (s, 1H, 1-OH); ${ }^{13} \mathrm{C}$ NMR (125 MHz, $\mathrm{CDCl}_{3}$ ): $\delta=16.6$ (q, C-6'), 20.3 (q, 3-CH $\left.\mathrm{CH}_{3}\right), 29.8$ (t, C-2'), 46.2 (d, C-3'), 59.6 (d, C-5'), 65.8 (t, CH ), 69.2 (C-4'), 96.7 (d, C-1'), 114.6 (s, C-1a), 121.4 (d, C-4), 126.9 (d, C-6), 127.4 (d, C-7), 130.6 (s, C-4a), 132.5 (s, C-2), 133.3 (s, C-9a), 133.6 (d, C-10a), 134.2 (s, C-6), 134.5 (d, C-7), 149.0 (s, C-3), 161.6 (C-1), 182.5 (s, C-9), 188.3 (s, C-10). EIMS (290 $\left.{ }^{\circ} \mathrm{C}\right)$ : $m / z(\%)=493(3)[\mathrm{M}+], 268$ (93); 252 (100), 239 (33), 165 (20), 113 (25). HREIMS: $m / z(\%)=$ 493.1348 (Calcd. for $\mathrm{C}_{24} \mathrm{H}_{22} \mathrm{~F}_{3} \mathrm{NO}_{7}, 493.1357$ ).

2-[O-(2,3,6-Trideoxy-3-trifluoroacetamido- $\alpha$-L-lyxo-hexopyranosyl)methyl]-1,4-dihydroxy9,10-anthraquinone (12). Aglycone 5 (2-hydroxymethyl-quinizarin) ${ }^{54,55}$ (78 mg, $0.29 \mathrm{mmol}$ ) was reacted as described in the general procedure to afford $94 \mathrm{mg}(66 \%)$ of the $\alpha$-glycoside 12; mp $230-231{ }^{\circ} \mathrm{C}$. IR: $3420(\mathrm{OH}, \mathrm{NH}), 1716,1696(\mathrm{C}=\mathrm{O}), 1620$ (quinone, chelated), $1588 \mathrm{~cm}^{-1}$ (arom.). ${ }^{1} \mathrm{H}$ NMR (270 MHz): $\delta=1.30\left(\mathrm{~d}, . J=6.7 \mathrm{~Hz}, 3 \mathrm{H}, 6^{\prime}-\mathrm{H}\right), 1.88(\mathrm{dt}, J=13.4,3.7 \mathrm{~Hz}, 1 \mathrm{H}$, $\left.2^{\prime} \mathrm{a}-\mathrm{H}\right), 2.13$ (dd, $\left.J=13.4,5.4,0.8 \mathrm{~Hz}, 1 \mathrm{H}, 2^{\prime} \mathrm{e}-\mathrm{H}\right), 3.67$ (m, 1H, 4'-H), 4.20 (q, $J=6.7 \mathrm{~Hz}, 1 \mathrm{H}, 5^{\prime}-$ H), 4.54 (m, 1H, 3'-H), 4.69 (dd, $J=14.5,1.1 \mathrm{~Hz}, 1 \mathrm{H}, 11-\mathrm{H}), 4.89$ (dd, $J=14.5,1.0 \mathrm{~Hz}, 11-\mathrm{H})$, 5.10 (br d, $\left.J=3.7 \mathrm{~Hz}, 1 \mathrm{H}, 1^{\prime}-\mathrm{H}\right), 7.49$ (s, 1H, 3-H), 7.86 (m, 2H, 6-H, 7-H), 8.36 (m, 2H, 5-H, 8$\mathrm{H}), 12.78(\mathrm{~s}, 1 \mathrm{H}, \mathrm{OH}), 13.31(\mathrm{~s}, 1 \mathrm{H}, \mathrm{OH})$. EIMS $\left(200^{\circ} \mathrm{C}\right): m / z(\%)=495(0.5)\left[\mathrm{M}^{+}\right], 300(100)$, 282 (35). Anal. Calcd for $\mathrm{C}_{23} \mathrm{H}_{20} \mathrm{NO}_{8} \mathrm{~F}_{3}$ (495.41): C, 55.76; H, 4.07; N, 2.83. Found: C, 55.50; H, $3.86 ; \mathrm{N}, 3.07$.

1-Hydroxy-8-methoxy-2-[1-O-(2',3',6'-trideoxy-3'-trifluoroacetamido-a-L-lyxo-hexopyranosyl)-methyl]-9,10-anthraquinone (13). Aglycone $6^{61}$ (255 mg, $\left.0.898 \mathrm{mmol}\right)$ was glycosylated 
as described in the general procedure (sugar chloride 2:567.6 mg, silver triflate: $510 \mathrm{mg}$ (1.98 mmol) to afford the $\alpha$-glycoside 13 (321.3 mg, 86.4\%); mp 229.5-231.5 ${ }^{\circ} \mathrm{C}$. IR: 3530, $3280(\mathrm{OH}$, $\mathrm{NH}), 2920(-\mathrm{CH}), 1700(\mathrm{CO}), 1670$ (quinone), 1630 (quinone, chelated), 1585, 1430, 1280, 1170, 1070, $745 \mathrm{~cm}^{-1} .{ }^{1} \mathrm{H}$ NMR $\left(500 \mathrm{MHz}, \mathrm{CDCl}_{3}\right): \delta=1.26\left(\mathrm{~d}, J=6.6 \mathrm{~Hz}, 3 \mathrm{H}, 66^{\prime}-\mathrm{H}\right), 1.85$ (ddd, $J=$ 13.6, 12.7, $\left.3.2 \mathrm{~Hz}, 1 \mathrm{H}, 2^{\prime} \mathrm{a}-\mathrm{H}\right), 1.92$ (d, $\left.J=8.6 \mathrm{~Hz}, 1 \mathrm{H}, 4^{\prime}-\mathrm{OH}\right), 2.08$ (dd, $J=13.6,4.9 \mathrm{~Hz}, 1 \mathrm{H}$, 2'e-H), 3.63 (d, $\left.J=8.6 \mathrm{~Hz}, 1 \mathrm{H}, 4^{\prime}-\mathrm{H}\right), 4.09$ (s, 3H, $\mathrm{OCCH}_{3}$ ), 4.16 (q, $\left.J=6.6 \mathrm{~Hz}, 1 \mathrm{H}, 5 '-\mathrm{H}\right), 4.51$ $\left(\mathrm{m}, 1 \mathrm{H}, 3^{\prime}-\mathrm{H}\right), 4.66\left(\mathrm{~d}, J=14.0 \mathrm{~Hz}, 1 \mathrm{H}, \mathrm{CH}_{2}\right), 4.89\left(\mathrm{~d}, J=14.0 \mathrm{~Hz}, 1 \mathrm{H}, \mathrm{CH}_{2}\right), 5.07$ (d, $J=3.2 \mathrm{~Hz}$, 1H, l'-H), 6.67 (d, $\left.J=8.4 \mathrm{~Hz}, 1 \mathrm{H}, 4^{\prime}-\mathrm{NH}\right), 7.38$ (dd, $\left.J=8.6,1.0 \mathrm{~Hz}, 1 \mathrm{H}, 7-\mathrm{H}\right), 7.77$ (dd, $J=8.6$, $7.7 \mathrm{~Hz}, 1 \mathrm{H}, 6-\mathrm{H}), 7.78$ (d, $J=7.7 \mathrm{~Hz}, 1 \mathrm{H}, 3-\mathrm{H}), 7.81$ (d, $J=7.7 \mathrm{~Hz}, 1 \mathrm{H}, 4-\mathrm{H}), 7.99$ (dd, $J=7.7$, $1.0 \mathrm{~Hz}, 1 \mathrm{H}, 5-\mathrm{H}), 13.32(\mathrm{~s}, 1 \mathrm{H}, 1-\mathrm{OH}) ;{ }^{13} \mathrm{C}$ NMR $\left(125 \mathrm{MHz}, \mathrm{DMSO}-\mathrm{d}_{6}\right): \delta=17.4$ (q, C-6'), 28.8 (t, C-2'), 47.4 (d, C-3'), 57.0 (q, $\left.\mathrm{OCH}_{3}\right), 63.0$ (d, C-5'), $66.8\left(\mathrm{t}, \mathrm{CH}_{2}\right), 67.2(\mathrm{C}-4$ '), 96.8 (d, C-1'), 115.2 (s, C-1a), 117.4 (s, C-10a), 118.3 (d, C-7), 119.7 (d, C-5), 119.8 (d, C-4), 131.7 (d, C-6), 134.4 (s, C-4a), 134.6 (s, C-9a), 136.9 (d, C-3), 159.4 (s, C-8), 161.1 (C-1), 182.2 (s, C-9), 188.8 $(\mathrm{s}, \mathrm{C}-10)$. EIMS $\left(300{ }^{\circ} \mathrm{C}\right): \mathrm{m} / z(\%)=509(0.1)[\mathrm{M}+], 284(51), 268$ (100), 250 (41), 113 (32). HREIMS: $m / z(\%)=509.1298\left(\right.$ Calcd. for $\mathrm{C}_{24} \mathrm{H}_{22} \mathrm{~F}_{3} \mathrm{NO}_{8}, 509.1298$ ).

\section{1,8-Dihydroxy-2-[1-O-(2',3',6'-trideoxy-3'-trifluoroacetamido- $\alpha$-L-lyxo-hexopyranosyl)-} methyl]-9,10-anthraquinone (14). Aglycone $7^{54}(300 \mathrm{mg}, 1.11 \mathrm{mmol})$ was glycosylated as described in the general procedure using $854 \mathrm{mg}(1.97 \mathrm{mmol})$ of halogenose 2 , and $614.1 \mathrm{mg}$ (2.39 mmol) of silver triflate; reaction time $1 \mathrm{~h} ; 17 \mathrm{mg}(0.063 \mathrm{mmol})$ of aglycone 7 was recovered (94\% conversion). Yield of $\alpha$-glycoside 14: $343.1 \mathrm{mg}(62.2 \%) \mathrm{mp} 254-255{ }^{\circ} \mathrm{C}$. IR: 3500, $3280(\mathrm{OH}, \mathrm{NH}), 2900(-\mathrm{CH}), 1700(\mathrm{CO}), 1670$ (quinone), 1625 (quinone, chelated), 1552, $1430,1285,1175,1073,748 \mathrm{~cm}^{-1}$. ${ }^{1} \mathrm{H}$ NMR $\left(500 \mathrm{MHz}, \mathrm{CDCl}_{3}\right): \delta=1.27$ (d, $J=6.6 \mathrm{~Hz}, 3 \mathrm{H}, 6^{6}-$ H), 1.88 (ddd, $J=12.6,3.6,2.5 \mathrm{~Hz}, 1 \mathrm{H}, 2$ 'a-H), 2.10 (dd, $J=12.6,5.2 \mathrm{~Hz}, 1 \mathrm{H}, 2$ 'e-H), 3.66 (br s, 1H, 4'-H), 4.16 (q, $J=6.6 \mathrm{~Hz}, 1 \mathrm{H}, 5$ '-H), 4.51 (dddd, $J=12.6,8.4,5.2,2.1 \mathrm{~Hz}, 1 \mathrm{H}, 3$ '-H), 4.65 $\left(\mathrm{d}, J=14.0 \mathrm{~Hz}, 1 \mathrm{H}, \mathrm{CH}_{2}\right), 4.88\left(\mathrm{~d}, J=14.0 \mathrm{~Hz}, 1 \mathrm{H}, \mathrm{CH}_{2}\right), 5.07$ (d, $\left.J=3.3 \mathrm{~Hz}, 1 \mathrm{H}, \mathrm{l}^{\prime}-\mathrm{H}\right), 6.70(\mathrm{~d}, J$ $\left.=8.4 \mathrm{~Hz}, 1 \mathrm{H}, 4^{\prime}-\mathrm{NH}\right), 7.70(\mathrm{t}, J=7.8 \mathrm{~Hz}, 1 \mathrm{H}, 6-\mathrm{H}), 7.32(\mathrm{~d}, J=7.8 \mathrm{~Hz}, 1 \mathrm{H}, 4-\mathrm{H}), 7.87-7.82(\mathrm{~m}$, $3 \mathrm{H}, 4-\mathrm{H}, 5-\mathrm{H}, 7-\mathrm{H}), 12.03(\mathrm{~s}, 1 \mathrm{H}, \mathrm{OH}), 12.42(\mathrm{~s}, 1 \mathrm{H}, \mathrm{OH}) ;{ }^{13} \mathrm{C}$ NMR $\left(125 \mathrm{MHz}, \mathrm{CDCl}_{3}\right): \delta=$ 16.6 (q, C-6'), 29.7 (t, C-2'), 46.2 (d, C-3'), 63.5 (d, C-5'), 65.9 (t, $\left.\mathrm{CH}_{2}\right), 69.1$ (C-4'), 96.7 (d, C1'), 114.6 (s, C-1a), 117.4 (s, C-10a), 119.8 (d, C-5), 120.1 (d, C-4), 124.6 (d, C-7), 132.7 (d, C6), 133.7 (s, C-4a), 133.8 (s, C-9a), 135.5 (d, C-3), 159.9 (s, C-8), 162.6 (C-1), 181.5 (s, C-9), 193.4 (s, C-10). EIMS $\left(300^{\circ} \mathrm{C}\right): m / z(\%)=495(2)[\mathrm{M}+], 270(95) ; 253$ (100), $226(28), 113$ (76). HREIMS: $m / z(\%)=495.1141$ (Calcd. for $\left.\mathrm{C}_{23} \mathrm{H}_{20} \mathrm{~F}_{3} \mathrm{NO}_{8}, 495.1141\right)$.

\section{1,5-Dimethoxy-4-hydroxy-3-[1-O-(2',3',6'-trideoxy-3'-trifluoroacetamido- $\alpha$-L-lyxo-hexo-}

pyranosyl)-methyl]-9,10-anthraquinone (15). The glycosylation of aglycone $\mathbf{8}^{59}(190 \mathrm{mg}$, $0.605 \mathrm{mmol})$ was performed as described in the general procedure using $571.8 \mathrm{mg}(1.32 \mathrm{mmol})$ of sugar chloride 2 , and $405 \mathrm{mg}$ of silver triflate $(1.57 \mathrm{mmol})$, reaction time $4 \mathrm{~h}$ : yields; recovered starting material 8, $1.3 \mathrm{mg}(0.034 \mathrm{mmol})$ (conversion 95\%); $\alpha$-glycoside 15, (168.3 $\mathrm{mg}, 54.5 \%) \mathrm{mp} 256-258{ }^{\circ} \mathrm{C}$; $\beta$-glycoside 21, $32 \mathrm{mg}(10.3 \%), \mathrm{mp} 263-265{ }^{\circ} \mathrm{C}$; and rearranged furanoside 24, $30 \mathrm{mg}$ (9.8 \%). IR: 3495, 3290, (OH, NH), 2980, 2940, 2850 (-CH), 1700 (CO), 1655 (quinone), 1630 (quinone, chelated), 1588, 1425, 1280, 1255, 1197, $765 \mathrm{~cm}^{-1} .{ }^{1} \mathrm{H}$ NMR 
$\left(500 \mathrm{MHz}, \mathrm{CDCl}_{3}\right): \delta=1.29\left(\mathrm{~d}, J=6.9 \mathrm{~Hz}, 3 \mathrm{H}, 6{ }^{\prime}-\mathrm{H}\right), 1.90\left(\mathrm{ddd}, J=13.0,12.5,3.6 \mathrm{~Hz}, 1 \mathrm{H}, 2^{\prime} \mathrm{a}-\right.$ H), 2.03 (br s, 1H, 4'-OH), 2.09 (dd, $\left.J=13.0,5.0 \mathrm{~Hz}, 1 \mathrm{H}, 2^{\prime} \mathrm{e}-\mathrm{H}\right), 3.66$ (dd, $J=8.0,2.5 \mathrm{~Hz}, 1 \mathrm{H}$, 4'-H), 4.05 (s, 3H, $\left.\mathrm{OCH}_{3}\right), 4.08\left(\mathrm{~s}, 3 \mathrm{H}, \mathrm{OCH}_{3}\right), 4.16$ (q, J=6.9 Hz, 1H, 5'-H), 4.54 (m, 1H, 3'-H), $4.67\left(\mathrm{dd}, J=14.9,1.2 \mathrm{~Hz}, 1 \mathrm{H}, \mathrm{CH}_{2}\right), 4.92\left(\mathrm{dd}, J=14.9,1.2 \mathrm{~Hz}, 1 \mathrm{H}, \mathrm{CH}_{2}\right), 5.08$ (d, $J=3.6 \mathrm{~Hz}$, $\left.1 \mathrm{H}, \mathrm{l}^{\prime}-\mathrm{H}\right), 6.76\left(\mathrm{~d}, J=8.4 \mathrm{~Hz}, 1 \mathrm{H}, 3^{\prime}-\mathrm{NH}\right), 7.30$ (dd, $\left.J=8.3,1.1 \mathrm{~Hz}, 1 \mathrm{H}, 6-\mathrm{H}\right), 7.51$ (s, 1H, 2-H), $7.75(\mathrm{dd}, J=8.3 \mathrm{~Hz}, J=8 \mathrm{~Hz}, 1 \mathrm{H}, 7-\mathrm{H}), 7.95(\mathrm{dd}, J=8.0,1.1 \mathrm{~Hz}, 8-\mathrm{H}), 13.52(\mathrm{~s}, 1 \mathrm{H}, \mathrm{OH}) ;{ }^{13} \mathrm{C}$ NMR (125 MHz, DMSO-d 6 ): $\delta=17.3$ (q, C-6'), 28.3 (t, C-2'), 47.3 (d, C-3'), 57.0 (q, OCH OC, $^{\prime}$ $57.1\left(\mathrm{q}, \mathrm{OCH}_{3}\right), 62.9\left(\mathrm{~d}, \mathrm{C}-5^{\prime}\right), 67.0\left(\mathrm{t}, \mathrm{CH}_{2}\right), 67.3\left(\mathrm{C}-4{ }^{\prime}\right), 96.8\left(\mathrm{~d}, \mathrm{C}-1^{\prime}\right), 115.2(\mathrm{~s}, \mathrm{C}-1 \mathrm{a}), 116.3$ (d, C-7), 117.5 (d, C-5), 117.7 (s, C-10a), 118.4 (d, C-3), 119.3 (s, C-4a), 136.6 (s, C-2), 136.9 (d, C-6), 136.6 (s, C-9a), 153.4 (s, C-1), 154.0 (s, C-4), 160.6 (s, C-8), 180.8 (s, C-9), 188.8 (s, C10). MS: $m / z(\%)=539(10)[\mathrm{M}+], 481$ (0.5), 418 (2), 384 (12), 314 (64), 265 (13), 251 (22), 193 (19), 137 (24), 121 (100), 57 (28). HREIMS: $m / z=539.1403$ (Calcd. for $\mathrm{C}_{25} \mathrm{H}_{24} \mathrm{~F}_{3} \mathrm{NO}_{9}$, 539.1403).

\section{1,4-Dihydroxy-5-methoxy-3-[1-O-(2',3',6'-trideoxy-3'-trifluoroacetamido- $\alpha$-L-lyxo-hexo-} pyranosyl)-methyl]-9,10-anthraquinone (16) and the isomeric rearranged furanoside 25. Aglycone $9^{59}$ (200 $\mathrm{mg}, 0.67 \mathrm{mmol}$ ) was glycosylated as described in the general procedure using sugar 2 (512 mg, $1.18 \mathrm{mmol})$ and $350 \mathrm{mg}(1.43 \mathrm{mmol})$ of silver triflate, reaction time 1h; yields: recovered aglycone 9 (25.5 mg, $0.085 \mathrm{mmol})$ (87\% conversion), $\alpha$-glycoside 16: $209.1 \mathrm{mg}$

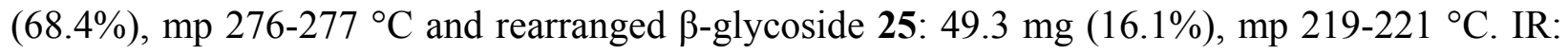
3530, $3270(\mathrm{OH}, \mathrm{NH}), 2920(-\mathrm{CH}), 1700(\mathrm{CO}), 1615,1580$ (quinone, chelated), 1445, 1425, 1295, 1252, 1190, 1155, $1057 \mathrm{~cm}^{-1} .{ }^{1} \mathrm{H} \mathrm{NMR}(400 \mathrm{MHz}): \delta=1.27$ (d, $\left.J=6.9 \mathrm{~Hz}, 3 \mathrm{H}, 6{ }^{\prime}-. \mathrm{H}\right)$, 1.88 (ddd, $\left.J=13.2,12.9,4.0 \mathrm{~Hz}, 1 \mathrm{H}, 2^{\prime}-\mathrm{a}-\mathrm{H}\right), 2.00$ (d, $\left.J=7.3 \mathrm{~Hz}, 1 \mathrm{H}, 4{ }^{\prime}-\mathrm{OH}\right), 2.12$ (dd, $J=13.2$, $\left.5.1 \mathrm{~Hz}, 1 \mathrm{H}, 2^{\prime} \mathrm{e}-\mathrm{H}\right), 3.66\left(\mathrm{dd}, J=7.3,3.5 \mathrm{~Hz}, 1 \mathrm{H}, 4^{\prime}-\mathrm{H}\right), 4.11\left(\mathrm{~s}, 3 \mathrm{H}, \mathrm{OCH}_{3}\right), 4.15$ (q, $J=6.9 \mathrm{~Hz}$, $1 \mathrm{H}, 5^{\prime}-\mathrm{H}$ ), 4.51 (dddd, $\left.J=12.9,8.0,5.1,3.5 \mathrm{~Hz}, 1 \mathrm{H}, 3^{\prime}-\mathrm{H}\right), 4.67$ (d, $J=15 \mathrm{~Hz}, 1 \mathrm{H}, \mathrm{CH}_{2}-\mathrm{Bridge}$ ), $4.87\left(\mathrm{~d}, J=15 \mathrm{~Hz}, 1 \mathrm{H}, \mathrm{CH}_{2}\right), 5.07$ (d, $\left.J=4 \mathrm{~Hz}, 1 \mathrm{H}, \mathrm{l}^{\prime}-\mathrm{H}\right), 6.74$ (d, $\left.J=8.0 \mathrm{~Hz}, 1 \mathrm{H}, 3^{\prime}-\mathrm{NH}\right), 7.26$ (s, 1H, 2-H), $7.40(\mathrm{dd}, J=8.9,1.0 \mathrm{~Hz}, 1 \mathrm{H}, 6-\mathrm{H}), 7.80(\mathrm{dd}, J=8.9,7.5 \mathrm{~Hz}, 1 \mathrm{H}, 7-\mathrm{H}), 8.05(\mathrm{~d}, J=$ $7.5 \mathrm{~Hz}, 1 \mathrm{H}, 8-\mathrm{H}), 12.90(\mathrm{~s}, 1 \mathrm{H}, \mathrm{OH}), 13.64(\mathrm{~s}, 1 \mathrm{H}, \mathrm{OH})$. EIMS $\left(180{ }^{\circ} \mathrm{C}\right): \mathrm{m} / z(\%)=525(18)$ $[\mathrm{M}+], 300$ (100), 282 (88), 271 (17), 113 (18). HREIMS: $m / z(\%)=525.1247$ (Calcd. for $\left.\mathrm{C}_{24} \mathrm{H}_{22} \mathrm{~F}_{3} \mathrm{NO}_{9}, 525.1247\right)$.

\section{1,4,5-Trihydroxy-3-[1-O-(2',3',6'-trideoxy-3'-trifluoroacetamido- $\alpha$-L-lyxo-hexopyranosyl)-} methyl]-9,10-anthraquinone (17). Aglycone 10 (250 $\mathrm{mg}, 0.87 \mathrm{mmol})$ was glycosylated as described in the general procedure using $640 \mathrm{mg}$ (1.47 mmol) of sugar 2 (450 $\mathrm{mg}, 1.75 \mathrm{mmol})$ and $250 \mathrm{mg}(0.87 \mathrm{mmol})$ of silver triflate; reaction time $1 \mathrm{~h}$. After chromatographic separation, $45.6 \mathrm{mg}(0.159 \mathrm{mmol})$ of aglycone 10 was recovered (conversion 82\%). Yield of 17, $195.7 \mathrm{mg}$ (53.6\%), mp 246-248 ${ }^{\circ} \mathrm{C}$; $\beta$-glycoside 22: $19.6 \mathrm{mg}$ (5.4\%), mp 227; rearranged furanoside $\beta$ glycoside 26: $42.9 \mathrm{mg}(11.7 \%)$, mp 205-207 ${ }^{\circ} \mathrm{C}$. Data for 17: IR, 3500, $\left.3420 \mathrm{OH}, \mathrm{NH}\right), 2980$, 2900 (-CH), 1715 (CO), 1605 (quinone, chelated), 1435, 1275, 1172,982, $770 \mathrm{~cm}^{-1} .{ }^{1} \mathrm{H} \mathrm{NMR}$ $\left(500 \mathrm{MHz}, \mathrm{CDCl}_{3}\right): \delta=1.25\left(\mathrm{~d}, J=6.4 \mathrm{~Hz}, 3 \mathrm{H}, 6^{\prime}-\mathrm{H}\right), 1.89$ (ddd, $J=13.0,12.8,3.5 \mathrm{~Hz}, 1 \mathrm{H}, 2^{\prime} \mathrm{a}-$ H), 1.95 (d, 8.2 Hz, 1H, 4'-OH), 2.11 (dd, J=13, 5.0 Hz, 1H, 2'e-H), 3.65 (br d, J=2 Hz, 1H, 4'H), 4.14 (q, $\left.J=6.4 \mathrm{~Hz}, 1 \mathrm{H}, 5^{\prime}-\mathrm{H}\right), 4.51\left(\mathrm{~m}, 1 \mathrm{H}, 3^{\prime}-\mathrm{H}\right), 4.65$ (d, $\left.J=14.7,1.5 \mathrm{~Hz}, 1 \mathrm{H}, \mathrm{CH}_{2}\right), 4.86$ 
(dd, $\left.J=14.7,1.5 \mathrm{~Hz}, 1 \mathrm{H}, \mathrm{CH}_{2}\right), 5.07$ (d, $\left.J=3.5 \mathrm{~Hz}, 1 \mathrm{H}, \mathrm{l}^{\prime}-\mathrm{H}\right), 6.69$ (d, $\left.J=7.9 \mathrm{~Hz}, 1 \mathrm{H}, 3^{\prime}-\mathrm{NH}\right)$, 7.31 (dd, $J=7.8,1.0 \mathrm{~Hz}, 1 \mathrm{H}, 6-\mathrm{H}), 7.45$ (s, 1H, 2-H), 7.71 (t, $J=7.8 \mathrm{~Hz}, 1 \mathrm{H}, 7-\mathrm{H}), 7.88$ (dd, $J=$ 7.8,1.0 Hz, 1H, 8-H), $12.16(\mathrm{~s}, 1 \mathrm{H}, \mathrm{OH}), 12.64(\mathrm{~s}, 1 \mathrm{H},-\mathrm{OH}), 13.03(\mathrm{~s}, 1 \mathrm{H},-\mathrm{OH}) ;{ }^{13} \mathrm{C}$ NMR $(125$ $\mathrm{MHz}_{\mathrm{CDCl}}$ ): $\delta=16.6$ (q, C-6'), 29.6 (t, C-2'), 46.1 (d, C-3'), 63.4 (d, C-6'), $66.0\left(\mathrm{~d}, \mathrm{CH}_{2}\right), 69.0$ (C-4'), 96.7 (d, C-1'), 111.9 (s, C-10a), 112.4 (s, C-4a), 119.5 (d, C-8), 124.6 (d, C-6), 126.1 (d, C-3), 127.0 (d, C-5), 127.1 (d, C-8), 133.2 (s, C-9a), 133.4 (d, C-2), 134.2 (d, C-7), 137.1 (d, C7), 155.4 (s, C-1), 158.3 (s, C-4), 162.7 (C-5), 185.9 (s, C-9), 191.4 (s, C-10). EIMS (260 ${ }^{\circ} \mathrm{C}$ ): $m / z(\%)=511(8)[\mathrm{M}+], 286(100) ; 269$ (82), 241 (24), 113 (18). HREIMS: $m / z(\%)=511.1090$ (Calcd. for $\left.\mathrm{C}_{23} \mathrm{H}_{20} \mathrm{~F}_{3} \mathrm{NO}_{9}, 511.1090\right)$.

\section{1-Hydroxy-3-methyl-2-[1-O-(2',3',6'-trideoxy-3'-trifluoroacetamido- $\beta$-L-lyxo-hexo-}

pyranosyl)-methyl]-9,10-anthraquinone (18). From the same reaction (see 11), $19.2 \mathrm{mg}(5.7 \%)$ of the more polar $\beta$-glycoside 18 was isolated, mp 250-251 ${ }^{\circ} \mathrm{C}$. IR: $3520,3285(\mathrm{OH}, \mathrm{NH}), 2990$, 2895 (-CH), 1700 (CO), 1670 (quinone), 1645 (quinone, chelated), 1595, 1560, 1365, 1292, 1187, 1176, 1060, $720 \mathrm{~cm}^{-1} .{ }^{1} \mathrm{H}-\mathrm{NHR}\left(500 \mathrm{MHz} \mathrm{CDCl}_{3}\right): \delta=1.35$ (d, $\left.J=6.5 \mathrm{~Hz}, 3 \mathrm{H}, 6{ }^{\prime}-\mathrm{H}\right), 1.58$ (ddd, $\left.J=13.0,11.0,10.0,1 \mathrm{H}, 2^{\prime} \mathrm{a}-\mathrm{H}\right), 2.06$ (ddd, $J=13.0,4.9,2.2 \mathrm{~Hz}, 1 \mathrm{H}, 2$ 'a-H), 2.14 (s, 1H, 4'-OH), 2.53 (s, 3H, 11-H), $3.53\left(\mathrm{~d}, J=9.7 \mathrm{~Hz}, 1 \mathrm{H}, 4^{\prime}-\mathrm{H}\right), 3.70$ (q, $\left.J=6.5 \mathrm{~Hz}, 1 \mathrm{H}, 5{ }^{\prime} \mathrm{H}\right), 4.11$ (m, 1H, 3'-H), 4.65 (dd, $\left.J=10.0,2.2 \mathrm{~Hz}, 1 \mathrm{H}, \mathrm{l}^{\prime} \mathrm{H}\right), 4.94\left(\mathrm{~d}, J=11.5 \mathrm{~Hz}, 1 \mathrm{H}, \mathrm{CH}_{2}\right), 5,02(\mathrm{~d}, J=11.5$ $\left.\mathrm{Hz}, 1 \mathrm{H}, \mathrm{CH}_{2}\right), 6.76$ (d, J=8.1 Hz, 1H, 3'-NH), 7.70 (s, 1H, 4-H), 7.82 (m, 2H, 6-H, 7-H), 8.33 (m, 2H, 5-H, 8-H), 13.13 (s, 1H, 1-OH); $\left.{ }^{13} \mathrm{C} \mathrm{NMR} \mathrm{(125} \mathrm{MHz,} \mathrm{CDCl}_{3}+\mathrm{CD}_{3} \mathrm{OD}\right): \delta=16.3$ (q, C6'), 20.0 (q, 3- $\left.\mathrm{CH}_{3}\right), 30.9$ (t, C-2'), 48.9 (d, C-3'), 60.7 (d, C-5'), 67.7 (t, $\left.\mathrm{CH}_{2}\right), 71.8\left(\mathrm{C}-4^{\prime}\right), 99.6$ (d, C-1'), 114.0 (s, C-1a), 121.3 (d, C-4), 126.8 (d, C-6), 127.2 (d, C-7), 130.3 (s, C-4a), 132.5 (s, C-2), 133.2 (s, C-9a), 133.4 (d, C-10a), 134.2 (s, C-6), 134.5 (d, C-7), 149.1 (s, C-3), 161.3 (C1), 182.7 (s, C-9), 188.2 (s, C-10). EIMS $\left(180{ }^{\circ} \mathrm{C}\right): m / z(\%)=493(0.1)\left[\mathrm{M}^{+}\right], 268(74), 252$ (100), 239 (27), 113 (12). HREIMS: $m / z(\%)=493.1347$ (Calcd. for $\mathrm{C}_{24} \mathrm{H}_{22} \mathrm{~F}_{3} \mathrm{NO}_{7}, 493.1348$ ).

\section{1,4-Dihydroxy-2-[O-(2,3,6-trideoxy-3-trifluoroacetamido- $\beta$-L-lyxo-hexopyranosyl)methyl]-}

9,10-anthraquinone (19). From the same reaction of aglycone 5 (see 12), $9 \mathrm{mg}(6 \%)$ of the more polar $\beta$-glycoside 19 was isolated; mp $244{ }^{\circ} \mathrm{C}$. IR: $3420(\mathrm{OH}, \mathrm{NH}) ; 1716,1690(\mathrm{C}=\mathrm{O})$; 1624 (quinone, chelated), $1588 \mathrm{~cm}^{-1}$ (aromat). ${ }^{1} \mathrm{H} \mathrm{NMR}\left(500 \mathrm{MHz}, \mathrm{CDCl}_{3}\right): \delta=1.34(\mathrm{~d}, J=6.7$ $\left.\mathrm{Hz}, 3 \mathrm{H}, 6{ }^{\prime}-\mathrm{H}\right), 1.74$ (dq, $\left.J=13.2,12.69 .5 \mathrm{~Hz}, 1 \mathrm{H}, 2 \mathrm{a}-\mathrm{H}\right), 2.23$ (ddd, $J=13.2,5.0,2.1 \mathrm{~Hz}, 1 \mathrm{H}$, 2'e-H), 3.56 (br d, $\left.J=4.0 \mathrm{~Hz}, 1 \mathrm{H}, 4^{\prime}-\mathrm{H}\right), 3.72$ (dq, $J=6.7,1.0 \mathrm{~Hz}, 1 \mathrm{H}, 5$ '-H), 4.18 (m, 1H, 3'-H), $4.71\left(\mathrm{dd}, J=10,2.5 \mathrm{~Hz}, 1 \mathrm{H}, 1^{\prime}-\mathrm{H}\right), 4.82$ (dd, $\left.J=15.0, J=1.0 \mathrm{~Hz}, 1 \mathrm{H}, 11 \mathrm{~b}-\mathrm{H}\right), 5.08$ (dd, $J=15.0$, $1.0 \mathrm{~Hz}, 1 \mathrm{H}, 11 \mathrm{a}-\mathrm{H}), 6.87$ (d, $J=7.8 \mathrm{~Hz}, 1 \mathrm{H}, \mathrm{NH}), 7.45$ (s, 1H, 3-H), 7.85 (m, 2H, 6-H, 7-H), $8.37(\mathrm{~m}, 2 \mathrm{H}, 5-\mathrm{H}, 8-\mathrm{H}), 12.90(\mathrm{~s}, 1 \mathrm{H},-\mathrm{OH}), 13.26(\mathrm{~s}, 1 \mathrm{H},-\mathrm{OH}) ;{ }^{13} \mathrm{C} \mathrm{NMR}\left(125 \mathrm{MHz}, \mathrm{CDCl}_{3}\right): \delta$ $=16.3\left(\mathrm{q}, \mathrm{C}-6^{\prime}\right), 29.7$ (t, C-2'), $49.1\left(\mathrm{~d}, \mathrm{C}-3^{\prime}\right), 65.0\left(\mathrm{~d}, \mathrm{C}-5^{\prime}\right), 68.4\left(\mathrm{~d}, \mathrm{C}-4^{\prime}\right), 71.5\left(\mathrm{~d}, \mathrm{C}-4^{\prime}\right), 99.8(\mathrm{~d}$, C-1'), 111.8 (s, C-10a), 112.4 (s, C-4a), 126.4 (d, C-5), 127.0 (d, C-8), 133.6 (s, C-9a), 133.7 (s, C-8a), 134.4 (d, C-7), 134.6 (d, C-6), 139.4 (d, C-3), 144.2 (s, C-2), 155.2 (s, C-4), 157.8 (s, C1), 186.1 (s, C-10), 187.3 (s, C-9). EIMS (180 $\left.{ }^{\circ} \mathrm{C}\right): \mathrm{m} / z(\%)=495(7)[\mathrm{M}+], 477(1), 421(05)$, 346 (0.5), 308 (0.5), 296 (2), 270 (100), 252 (71), 225 (18), 196 (7), 168 (5), 140 (7), 113 (13), 86 (10). HREIMS: $m / z=495.1143$ (Calcd. for $\mathrm{C}_{29} \mathrm{H}_{28} \mathrm{~F}_{3} \mathrm{NO}_{12}, 495.1141$ ). 


\section{1-Hydroxy-8-methoxy-2-[1-O-(2',3',6'-trideoxy-3'-trifluoroacetamido- $\beta$-L-lyxo-hexo-}

pyranosyl)-methyl]-9,10-anthraquinone (20). From the same reaction (see 13), the corresponding $\beta$-glycoside 20 was isolated; yield, $29 \mathrm{mg}$ (7.8\%), $\mathrm{mp} 249.5-251.5{ }^{\circ} \mathrm{C}$. IR: 3550 , $3290(\mathrm{OH}, \mathrm{NH}), 2920(-\mathrm{CH}), 1710(\mathrm{CO}), 1665$ (quinone), 1630 (quinone, chelated), 1580, 1430, 1277, 1170, 1070,745 $\mathrm{cm}^{-1} .{ }^{1} \mathrm{H}$ NMR $\left(500 \mathrm{MHz}, \mathrm{CDCl}_{3}\right): \delta=1.35\left(\mathrm{~d}, J=6.5 \mathrm{~Hz}, 3 \mathrm{H}, 6{ }^{\prime}-\mathrm{H}\right), 1.70$ (ddd, $\left.J=13.0,12.5,9.7 \mathrm{~Hz}, 1 \mathrm{H}, 2^{\prime} \mathrm{a}-\mathrm{H}\right), 2,20$ (ddd, $J=12.9,4.9,2.2 \mathrm{~Hz}, 1 \mathrm{H}, 2$ 'e-H), 3.54 (br s, $\left.1 \mathrm{H}, 4^{\prime}-\mathrm{H}\right), 3.69$ (q, $\left.J=6.5 \mathrm{~Hz}, 1 \mathrm{H}, 5^{\prime}-\mathrm{H}\right), 4.17$ (m, 1H, 3'-H), 4.09 (s, 3H, OCH $), 4.70$ (dd, $J=$ 10.0, 2.2 Hz, 1H, l'-H), 4.84 (d, $\left.J=14.2 \mathrm{~Hz}, 1 \mathrm{H}, \mathrm{CH}_{2}\right), 5.06$ (d, $\left.J=14.2 \mathrm{~Hz}, 1 \mathrm{H}, \mathrm{CH}_{2}\right), 6.80$ (d, $J$ $=8.5 \mathrm{~Hz}, 1 \mathrm{H}, 3$ '-NH), 7.37 (dd, $J=7.5,1.0 \mathrm{~Hz}, 1 \mathrm{H}, 7-\mathrm{H}), 7.75(\mathrm{~d}, J=7.8 \mathrm{~Hz}, 1 \mathrm{H}, 3-\mathrm{H}), 7.77$ (t, $J=7.5 \mathrm{~Hz}, 1 \mathrm{H}, 6-\mathrm{H}), 7.79$ (d, $J=7.8 \mathrm{~Hz}, 1 \mathrm{H}, 4-\mathrm{H}), 7.99$ (dd, $J=7.7 \mathrm{~Hz}, 1.0 \mathrm{~Hz}, 1 \mathrm{H}, 5-\mathrm{H}), 13.32$ $(\mathrm{s}, 1 \mathrm{H}, 1-\mathrm{OH}) ;{ }^{13} \mathrm{C}$ NMR $\left(125 \mathrm{MHz}, \mathrm{CDCl}_{3}\right): \delta=16.6$ (q, C-6'), 31.5 (t, C-2'), 49.2 (d, C-3'), 56.7 $\left(\mathrm{q}, \mathrm{OCH}_{3}\right), 65.3\left(\mathrm{~d}, \mathrm{C}-5^{\prime}\right), 68.5\left(\mathrm{t}, \mathrm{CH}_{2}\right), 71.5(\mathrm{C}-4$ '), 99.8 (d, C-1'), 116.4 (s, C-1a), 118.2 (d, C7), 118.5 (s, C-10a), 120.2 (d, C-5), 120.8 (d, C-4), 131.7 (d, C-6), 133.8 (s, C-4a), 134.0 (s, C9a), 135.9 (d, C-3), 159.7 (s, C-8), 161.0 (C-1), 182.5 (s, C-9), 189.2 (s, C-10). EIMS (180 $\left.{ }^{\circ} \mathrm{C}\right)$ : $m / z(\%)=509(4)[\mathrm{M}+], 284$ (91), $268(100), 250$ (23). HREIMS: $m / z(\%)=509.1298$ (Calcd. for $\mathrm{C}_{24} \mathrm{H}_{22} \mathrm{~F}_{3} \mathrm{NO}_{8}, 509.1298$ ).

\section{1,5-Dimethoxy-4-hydroxy-3-[1-O-(2',3',6'-Trideoxy-3'-trifluoroacetamido- $\beta$-L-lyxo-hexo-}

pyranosyl)-methyl]-9,10-anthraquinone (21). From the same reaction of aglycone $\mathbf{8}^{60}$ (see 15) (190 mg, $0.605 \mathrm{mmol}$ ), the $\beta$-glycoside 21 (32 mg, 10.3\%) was isolated; mp 263-265 ${ }^{\circ} \mathrm{C}$. IR: 3420, $3290(\mathrm{OH}, \mathrm{NH}), 2940$ (-CH), 1705 (CO), 1665 (quinone), 1630 (quinone, chelated), 1585, 1425, 1250, $1160 \mathrm{~cm}^{-1} .{ }^{1} \mathrm{H}$ NMR (400 MHz, $\left.\mathrm{CDCl}_{3}\right): \delta=1.30$ (d, $J=6.5 \mathrm{~Hz}, 3 \mathrm{H}, 6$ '-H), 1.75 (ddd, $\left.J=13.0,12.5,9.8 \mathrm{~Hz}, 1 \mathrm{H}, 2^{\prime} \mathrm{a}-\mathrm{H}\right), 2.14$ (d, $\left.J=9.7 \mathrm{~Hz}, 1 \mathrm{H}, 4^{\prime}-\mathrm{OH}\right), 2.28$ (ddd, $J=13.0,2.3$, $\left.4.2 \mathrm{~Hz}, 1 \mathrm{H}, 2^{\prime} \mathrm{e}-\mathrm{H}\right), 3.58$ (d, $\left.J=9.7 \mathrm{~Hz}, 1 \mathrm{H}, 4^{\prime}-\mathrm{H}\right), 3.73$ (q, $\left.J=6.5 \mathrm{~Hz}, 1 \mathrm{H}, 5 '-\mathrm{H}\right), 4.63$ (s, 3H, $\left.\mathrm{OCH}_{3}\right), 4.08\left(\mathrm{~s}, 3 \mathrm{H}, \mathrm{OCH}_{3}\right), 4.16\left(\mathrm{ddd}, J=12.5,8.3,4.2 \mathrm{~Hz}, 1 \mathrm{H}, 3^{\prime}-\mathrm{H}\right), 4.73$ (dd, $J=9.8,2.3 \mathrm{~Hz}$, $\left.1 \mathrm{H}, \mathrm{l}^{\prime}-\mathrm{H}\right), 4.32$ (d, $\left.J=14.9 \mathrm{~Hz}, 1 \mathrm{H}, \mathrm{CH}_{2}\right), 5.11$ (d, $\left.J=14.9 \mathrm{~Hz}, 1 \mathrm{H}, \mathrm{CH}_{2}\right), 6.86(\mathrm{~d}, J=8.3 \mathrm{~Hz}$, $\left.1 \mathrm{H}, 3^{\prime}-\mathrm{NH}\right), 7.44$ (dd, $\left.J=8.8,1.0 \mathrm{~Hz}, 1 \mathrm{H}, 6-\mathrm{H}\right), 7.50$ (s, 1H, 2-H), 7.75 (dd, $J=8.8,8.0 \mathrm{~Hz}, 1 \mathrm{H}$, 7-H), 7.98 (dd, $J=8.0,1.0 \mathrm{~Hz}, 1 \mathrm{H}, 8-\mathrm{H}), 13.51$ (s, 1H, 4-OH). HREIMS: $m / z=539.1401$ (Calcd. for $\mathrm{C}_{25} \mathrm{H}_{24} \mathrm{~F}_{3} \mathrm{NO}_{9}, 539.1403$ ).

\section{1,4,5-Trihydroxy-3-[1-O-(2',3',6'-trideoxy-3'-trifluoroacetamido- $\beta$-L-lyxo-hexopyranosyl)-} methyl]-9,10-anthraquinone (22). (See reaction for 17). ${ }^{1} \mathrm{H}$ NMR $\left(500 \mathrm{MHz}, \mathrm{CDCl}_{3}\right): \delta=1.35$ (d, $\left.J=6.4 \mathrm{~Hz}, 3 \mathrm{H}, 6^{\prime}-\mathrm{H}\right), 1.75$ (ddd, $\left.J=13.0,12.8,3.5 \mathrm{~Hz}, 1 \mathrm{H}, 2^{\prime} \mathrm{a}-\mathrm{H}\right), 2.07$ (d, $J=8.2 \mathrm{~Hz}, 1 \mathrm{H}$, 4'-OH), 2.20 (dd, $J=13.0,5.0 \mathrm{~Hz}, 1 \mathrm{H}, 2$ 'e-H), 3.56 (br d, $\left.J=2 \mathrm{~Hz}, 1 \mathrm{H}, 4{ }^{\prime}-\mathrm{H}\right), 3.72$ (q, $J=6.4$ Hz, 1H, 5'-H), 4.20 (m, 1H, 3'-H), 4.71 (dd, $J=10.0,2.5$ Hz, 1H, l'-H), 4.80 (d, J=14.7, $1.5 \mathrm{~Hz}$, $\left.1 \mathrm{H}, \mathrm{CH}_{2}\right), 5.07\left(\mathrm{dd}, J=14.7,1.5 \mathrm{~Hz}, 1 \mathrm{H}, \mathrm{CH}_{2}\right), 6.82(\mathrm{~d}, J=7.9 \mathrm{~Hz}, 1 \mathrm{H}, 3 \mathrm{\prime}-\mathrm{NH}), 7.31(\mathrm{dd}, J=$ 7.8,1.0 Hz, 1H, 6-H), $7.46(\mathrm{~s}, 1 \mathrm{H}, 2-\mathrm{H}), 7.71(\mathrm{t}, J=7.8 \mathrm{~Hz}, 1 \mathrm{H}, 7-\mathrm{H}), 7.89$ (dd, $J=7.8,1.0 \mathrm{~Hz}$, $1 \mathrm{H}, 8-\mathrm{H}), 12.15(\mathrm{~s}, 1 \mathrm{H}, \mathrm{OH}), 12.62(\mathrm{~s}, 1 \mathrm{H},-\mathrm{OH}), 13.01(\mathrm{~s}, 1 \mathrm{H},-\mathrm{OH}) ;{ }^{13} \mathrm{C} \mathrm{NMR}(125 \mathrm{MHz}$, $\left.\mathrm{CDCl}_{3}\right): \delta=16.6\left(\mathrm{q}, \mathrm{C}-6\right.$ '), 29.7 (t, C-2'), 49.1 (d, C-3'), 65.0 (d, C-6'), $68.5\left(\mathrm{~d}, \mathrm{CH}_{2}\right), 71.5\left(\mathrm{C}-4^{\prime}\right)$, 99.9 (d, C-1'), 111.7 (s, C-10a), 112.0 (s, C-4a), 119.5 (d, C-8), 124.6 (d, C-6), 127.0 (d, C-5, C8), 133.2 (s, C-9a), 133.7 (d, C-6, C-7), 134.2 (d,), 137.1 (d, C-3), 155.2 (s, C-1), 158.3 (s, C-4), 162.7 (C-5), 185.9 (s, C-9), 191.4 (s, C-10). EIMS (180 $\left.{ }^{\circ} \mathrm{C}\right): m / z(\%)=525$ (19) [M+], 451 (2), 
418 (1), 368 (1), 312 (4), 300 (100), 282 (83), 266 (20), 255 (17), 238 (10), 169 (8), 155 (10), 140 (16), 86 (24). HREIMS: $m / z=525.1247$ (Calcd. for $\mathrm{C}_{24} \mathrm{H}_{22} \mathrm{~F}_{3} \mathrm{NO}_{9}, 525.1247$ ).

\section{1,8-Dihydroxy-2-[1-O-(2',3',6'-Trideoxy-3'-trifluoroacetamido-a-L-lyxo-hexofuranosyl)-}

methyl]-9,10-anthraquinone (23). From the same reaction (see 14) was isolated the rearranged $\beta$-glycoside 23, yield: $37.7 \mathrm{mg}$ (7.3\%), mp 191-193 ${ }^{\circ} \mathrm{C}$. IR: 3400, 3290 (OH, NH), 2980, 2920 ($\mathrm{CH}$ ), 1710 (CO), 1670 (quinone), 1625 (quinone, chelated), 1560, 1430, 1285, 1182, 1070, 743 $\mathrm{cm}^{-1} .{ }^{1} \mathrm{H}$ NMR $\left(500 \mathrm{MHz}, \mathrm{CDCl}_{3}\right): \delta=1.30\left(\mathrm{~d}, J=6 \mathrm{~Hz}, 3 \mathrm{H}, 66^{\prime}-\mathrm{H}\right), 2.00\left(\mathrm{~d}, J=5.5 \mathrm{~Hz}, 1 \mathrm{H}, 5^{\prime}-\right.$ $\mathrm{OH}), 2.04\left(\mathrm{~d}, J=13.5 \mathrm{~Hz}, 1 \mathrm{H}, 2^{\prime} \mathrm{a}-\mathrm{H}\right), 2.30$ (ddd, $\left.J=13.5,7.7,4.2 \mathrm{~Hz}, 1 \mathrm{H}, 2^{\prime} \mathrm{e}-\mathrm{H}\right), 3.84$ (ddq, $J=$ 5.5, 6.0, 4.8 Hz, 1H, 5'-H), 3.88 (br d, $J=4.8 \mathrm{~Hz}, 1 \mathrm{H}, 4^{\prime}-\mathrm{H}$ ), 4.52 (br d, $J=7.7 \mathrm{~Hz}, 1 \mathrm{H}, 3^{\prime}-\mathrm{H}$ ), $4.57\left(\mathrm{~d}, J=12.2 \mathrm{~Hz}, 1 \mathrm{H}, \mathrm{CH}_{2}\right) 5.01\left(\mathrm{~d}, J=12.2 \mathrm{~Hz}, 1 \mathrm{H}, \mathrm{CH}_{2}\right), 5.45\left(\mathrm{~d}, J=4.2 \mathrm{~Hz}, 1 \mathrm{H}, \mathrm{l}^{\prime}-\mathrm{H}\right), 7.30$ $(\mathrm{d}, J=8.7 \mathrm{~Hz}, 1 \mathrm{H}, 3-\mathrm{H}), 7.39$ (d, $J=8.0 \mathrm{~Hz}, 1 \mathrm{H}, 3$ '-NH), 7.73 (m, 2H, 6-H, 7-H), 7.86 (m, 2H, 4-H, 5-H), $11.98(\mathrm{~s}, 1 \mathrm{H}, \mathrm{OH}), 12.58(\mathrm{~s}, 1 \mathrm{H}, \mathrm{OH}) ;{ }^{13} \mathrm{C} \mathrm{NMR}\left(125 \mathrm{MHz}, \mathrm{CDCl}_{3}\right): \delta=16.6(\mathrm{q}, \mathrm{C}-$ 6'), 38.9 (t, C-2'), 50.9 (d, C-3'), 64.1 (d, C-5'), 68.4 (t, $\left.\mathrm{CH}_{2}\right), 89.8$ (C-4'), 103.9 (d, C-1'), 115.7 (s, C-1a), 115.8 (s, C-10a), 117.0 (d, C-7), 119.7 (d, C-5), 120.2 (d, C-4), 124.6 (d, C-7), 133.1 (d, C-6), 133.6 (s, C-4a), 136.5 (s, C-9a), 137.5 (d, C-3), 160.5 (s, C-8), 162.7 (C-1), 181.4 (s, C9), 193.3 (s, C-10). EIMS (180 $\left.{ }^{\circ} \mathrm{C}\right): m / z(\%)=495$ (2) [M+], 415, (2), 344 (5), 312 (6), 270 (42), 253 (100), 224 (27), 113 (58). HREIMS: $m / z(\%)=495.1141$ (Calcd. for $\mathrm{C}_{23} \mathrm{H}_{20} \mathrm{~F}_{3} \mathrm{NO}_{8}$, 495.1141).

\section{1,5-Dimethoxy-4-hydroxy-3-[1-O-(2',3',6'-trideoxy-3'-trifluoroacetamido- $\beta$-L-lyxo-hexo-}

furanosyl)-methyl]-9,10-anthraquinone (24). From the same reaction of aglycone $\mathbf{8}^{60}$ (see 15) the furanosyl-glycoside 24 (29 mg, 9.3\%) was isolated. $\mathrm{mp} 226.227{ }^{\circ} \mathrm{C}$. IR: $3420,3290(\mathrm{OH}$, $\mathrm{NH}), 2940$ (-CH), 1705 (CO), 1665 (quinone), 1630 (quinone, chelated), 1585, 1425, 1250, 1160 $\mathrm{cm}^{-1} .{ }^{1} \mathrm{H}$ NMR $\left(500 \mathrm{MHz}, \mathrm{CDCl}_{3}\right): \delta=1.28\left(\mathrm{~d}, J=6.5 \mathrm{~Hz}, 3 \mathrm{H}, 6{ }^{\prime}-\mathrm{H}\right), 2.05(\mathrm{~d}, J=13.9 \mathrm{~Hz}, 1 \mathrm{H}$, 2'a-H), 2.32 (ddd, $J=13.9,7.9,4.2 \mathrm{~Hz}, 1 \mathrm{H}, 2$ 'b-H), 3.84 (dq, $\left.J=6.5,5.0 \mathrm{~Hz}, 1 \mathrm{H}, 5^{\prime}-\mathrm{H}\right), 3.88$ (dd, $\left.J=2.1 \mathrm{~Hz}, J=5.0 \mathrm{~Hz}, 1 \mathrm{H}, 4^{\prime}-\mathrm{H}\right), 4.03\left(\mathrm{~s}, 3 \mathrm{H}, \mathrm{OCH}_{3}\right), 4.09\left(\mathrm{~s}, 3 \mathrm{H}, \mathrm{OCH}_{3}\right), 4.55(\mathrm{dd}, J=8.3,2.1$ $\mathrm{Hz}, 1 \mathrm{H}, 3 \mathrm{\prime}-\mathrm{H}), 4.58$ ( d, $\left.J=13.7 \mathrm{~Hz}, 1 \mathrm{H}, \mathrm{CH}_{2}\right), 4.99$ (d, $\left.J=13.7 \mathrm{~Hz}, 1 \mathrm{H}, \mathrm{CH}_{2}\right), 5.44(\mathrm{~d}, J=4.2$ $\left.\mathrm{Hz}, 1 \mathrm{H}, 1^{\prime}-\mathrm{H}\right), 7.21(\mathrm{dd}, J=7.8 \mathrm{~Hz}, 1.5 \mathrm{~Hz}, 1 \mathrm{H}, 7-\mathrm{H}), 7.27$ (s, 1H, 2-H), 7.38 (br d, $J=8.3 \mathrm{~Hz}$, 1H, 3'-NH), 7.74 (t, $J=7.8 \mathrm{~Hz}, 1 \mathrm{H}, 6-\mathrm{H}), 7.96$ (dd, $J=7.8,1.5 \mathrm{~Hz}, 1 \mathrm{H}, 5-\mathrm{H}), 13.68$ (s, 1H, OH). ${ }^{13} \mathrm{C}$ NMR (50 MHz, $\mathrm{CDCl}_{3}$ ): $\delta=19.4$ (q, C-6'), 39.1 (t, C-2'), 50.9 (d, C-3'), 56.9 .0 (q, $\mathrm{OCH}_{3}$ ), 57.0 (q, $\mathrm{OCH}_{3}$ ), 64.4 (d, C-5'), 68.4 (t, $\mathrm{CH}_{2}$ ), 89.7 (C-4'), 104.0 (d, C-1'), 116.8 (s, C-1a), 117.0 (d, C-7), 118.8 (d, C-5), 120.0 (s, C-10a), 120.1 (d, C-3), 121.5 (s, C-4a), 135.2 (s, C-2), 135.9 (d, C-6), 137.4 (s, C-9a), 153.3 (s, C-1), 154.9 (s, C-4), 160.5 (s, C-8), 180.6 (s, C-9), 189.1 (s, C-10). MS: $m / z(\%)=539(19)[\mathrm{M}+], 521$ (0.5), 465 (0.5), 418 (0.5), $384(0.5), 314$ (100), 297 (39), 239 (12), 209 (6), 140 (7), 121 (8), 69 (13). HREIMS: $m / z=539.1398$ (Calcd. for $\left.\mathrm{C}_{25} \mathrm{H}_{24} \mathrm{~F}_{3} \mathrm{NO}_{9}, 539.1403\right)$.

\section{1,4-Dihydroxy-5-methoxy-3-[1-O-(2',3',6'-trideoxy-3'-trifluoroacetamido- $\beta$-L-lyxo-hexo-}

furanosyl)-methyl]-9,10-anthraquinone (25). From the same reaction (see 16) the rearranged glycoside 25 was isolated. Yield $49.3 \mathrm{mg}(16.1 \%), \mathrm{mp} 219-221^{\circ} \mathrm{C}$. IR: $3540,3315(\mathrm{OH}, \mathrm{NH})$, 2940 (-CH), 1695 (CO), 1617, 1580 (quinone, chelated), 1440, 1255, 1180, 1053, $732 \mathrm{~cm}^{-1} .{ }^{1} \mathrm{H}$ NMR (400 MHz): $\delta=1.28$ (d, $\left.J=6.5 \mathrm{~Hz}, 3 \mathrm{H}, 66^{\prime}-\mathrm{H}\right), 2.05$ (d, $J=13.9 \mathrm{~Hz}, 1 \mathrm{H}, 2$ 'a-H), 2.32 (ddd, 
$\left.J=13.9,7.9,4.2 \mathrm{~Hz}, 1 \mathrm{H}, 2^{\prime}-\mathrm{He}\right), 3.84(\mathrm{dq}, J=6.5,5.0 \mathrm{~Hz}, 1 \mathrm{H}, 5 '-\mathrm{H}), 3.88(\mathrm{dd}, J=2.1 \mathrm{~Hz}, J=$ $\left.5.0 \mathrm{~Hz}, 1 \mathrm{H}, 4^{\prime}-\mathrm{H}\right), 4.09$ (s, 3H, $\left.\mathrm{OCH}_{3}\right), 4.55\left(\mathrm{dd}, J_{3^{\prime}, 4^{\prime}}=8.3,2.1 \mathrm{~Hz}, 1 \mathrm{H}, 3{ }^{\prime}-\mathrm{H}\right), 4.58$ ( d, $J=13.7$ $\left.\mathrm{Hz}, 1 \mathrm{H}, \mathrm{CH}_{2}\right), 4.99$ (d, J=13.7 Hz, 1H, $\left.\mathrm{CH}_{2}\right), 5.44$ (d, J=4.2 Hz, 1H, 1'-H), 7.27 (s, 1H, 2-H), 7.38 (dr d, $\left.J=8.3 \mathrm{~Hz}, 1 \mathrm{H}, 3^{\prime}-\mathrm{NH}\right), 7.42$ (dd, $\left.J=8.9 \mathrm{~Hz}, 1.0 \mathrm{~Hz}, 1 \mathrm{H}, 6-\mathrm{H}\right), 7.81$ (dd, $J=8.9,7.8$ $\mathrm{Hz}, 1 \mathrm{H}, 7-\mathrm{H}), 8.08$ (dd, $J=7.8,1.8 \mathrm{~Hz}, 1 \mathrm{H}, 8-\mathrm{H}), 12.86$ (s, 1H, OH), 13.75 (s, 1H, OH). MS: m/e $=525\left(2 / \mathrm{M}^{+}\right) ; 524$ (11, M-H $\left.{ }^{+}\right) ; 300$ (100), 284 (42), 283 (68), 282 (62), 271 (13), 240 (4). HREIMS: $m / z=525.1243$ (Calcd. for $\mathrm{C}_{24} \mathrm{H}_{22} \mathrm{~F}_{3} \mathrm{NO}_{9}, 525.1247$ ).

\section{1,4,5-Trihydroxy-3-[1-O-(2',3',6'-trideoxy-3'-trifluoroacetamido- $\beta$-L-lyxo-hexofuranosyl)-} methyl]-9,10-anthraquinone (26) (see 17). IR: 3420, $3290(\mathrm{OH}, \mathrm{NH}), 2980,2930(-\mathrm{CH}), 1705$ (CO), 1605 (quinone, chelated), 1435, 1255, 1178, $725 \mathrm{~cm}^{-1} .{ }^{1} \mathrm{H}$ NMR $\left(400 \mathrm{MHz}, \mathrm{CDCl}_{3}\right): \delta=$ 1.29 (d, $\left.J=6.5 \mathrm{~Hz}, 3 \mathrm{H}, 66^{\prime}-\mathrm{H}\right), 1.97$ (d, $\left.J=5.7 \mathrm{~Hz}, 1 \mathrm{H}, 55^{\prime}-\mathrm{OH}\right), 2.06$ (d, $\left.J=14 \mathrm{~Hz}, 1 \mathrm{H}, 2^{\prime}-\mathrm{H}\right)$, 2.33 (ddd, $\left.J=14.0,7.7,4.2 \mathrm{~Hz}, 1 \mathrm{H}, 2^{\prime} \mathrm{e}-\mathrm{H}\right), 3.85$ (ddd, $\left.J_{4}{ }^{\prime}, 5^{\prime}=6.5,5.7,5.0,1 \mathrm{H}, 5^{\prime}-\mathrm{H}\right), 3.89$ (dd, $J$ $\left.=5.0,2.0 \mathrm{~Hz}, 1 \mathrm{H}, 4^{\prime}-\mathrm{H}\right), 4.56\left(\mathrm{~d}, J=14 \mathrm{~Hz}, 1 \mathrm{H}, \mathrm{CH}_{2}\right), 4.58$ (br d, $\left.J=\mathrm{Hz}, 1 \mathrm{H}, 3^{\prime}-\mathrm{H}\right), 5.00$ (d, $J=$ $\left.14.0 \mathrm{~Hz}, 1 \mathrm{H}, \mathrm{CH}_{2}\right), 5.45$ (d, $\left.J=4.0 \mathrm{~Hz}, 1 \mathrm{H}, 1^{\prime}-\mathrm{H}\right), 7.39$ (br d, $\left.J=8.0 \mathrm{~Hz}, 1 \mathrm{H}, 3^{\prime}-\mathrm{NH}\right), 7.32-7.92$ $(\mathrm{m}, 4 \mathrm{H}$, aromatic $\mathrm{H}), 12.12(\mathrm{~s}, 1 \mathrm{H}, \mathrm{OH}), 12.78(\mathrm{~s}, 1 \mathrm{H}, \mathrm{OH}), 13.00(\mathrm{~s}, 1 \mathrm{H}, \mathrm{OH})$. EIMS $\left(180{ }^{\circ} \mathrm{C}\right)$ : $m / z(\%)=511(6)[\mathrm{M}+], 467$ (1), 437 (3), 354 (2), 324 (3), 286 (96), 270 (100), 241 (24), 213 (10), 180 (10), 169 (18), 155 (24), 140 (40), 113 (20), 86 (75). HREIMS: $m / z=511.1089$ (Calcd. for $\left.\mathrm{C}_{23} \mathrm{H}_{20} \mathrm{~F}_{3} \mathrm{NO}_{12}, 511.1090\right)$.

1,4-Dihydroxy-2-[1-O-(2,3,6-trideoxy-3-trifluoroacetamido-a-L-lyxo-hexopyranosyl)-butyl]9,10-anthraquinone (nonpolar) (28). The glycosylation was performed as described in the general procedure starting with $51 \mathrm{mg}(0.16 \mathrm{mmol})$ of aglycone $27^{62}$ to afford $23 \mathrm{mg}(26 \%)$ of the less polar glycoside, mp $225^{\circ} \mathrm{C}$. IR: $3450(\mathrm{OH}, \mathrm{NH}), 1710(\mathrm{C}=\mathrm{O}), 1621$ (quinone, chelated), 1586 (arom.) $\mathrm{cm}^{-1} .{ }^{1} \mathrm{H}$ NMR $\left(500 \mathrm{MHz}, \mathrm{CDCl}_{3}\right): \delta=0.89$ (d, $J=6.6 \mathrm{~Hz}, 3 \mathrm{H}, 6$ '-H), 0.94 (t, $J=$ $\left.7.4 \mathrm{~Hz}, 3 \mathrm{H}, \mathrm{CH}_{2} \mathrm{CH}_{3}\right), 1.30-1.47\left(\mathrm{~m}, 2 \mathrm{H}, \mathrm{CH}_{2}\right), 1.70-1.86\left(\mathrm{~m}, 2 \mathrm{H}, \mathrm{CH}_{2}\right), 1.82$ (dt, $J=13.2 \mathrm{~Hz}, J$ $\left.=4.0 \mathrm{~Hz}, 1 \mathrm{H}, 2^{\prime} \mathrm{b}-\mathrm{H}\right), 1.85(\mathrm{~d}, J=8.0 \mathrm{~Hz}, 1 \mathrm{H},-\mathrm{OH}), 2.08\left(\mathrm{dd}, J=13.2, J=5.2 \mathrm{~Hz}, 1 \mathrm{H}, 2^{\prime} \mathrm{a}-\mathrm{H}\right)$, 3.54 (br d, $\left.J=6.0 \mathrm{~Hz}, 1 \mathrm{H}, 4^{\prime}-\mathrm{H}\right), 3.85$ (br q, $\left.J=6.4 \mathrm{~Hz}, 1 \mathrm{H}, 5^{\prime}-\mathrm{H}\right), 4.42$ (m, 1H, 3'-H), 5.10 (d, $J$ $\left.=3.8 \mathrm{~Hz}, 1 \mathrm{H}, 1^{\prime}-\mathrm{H}\right), 5.13\left(\mathrm{dd}, J=7.2, J=5.3 \mathrm{~Hz}, 1 \mathrm{H}, \mathrm{CHCH}_{2}\right), 6.69(\mathrm{br} \mathrm{d}, J=8.0 \mathrm{~Hz}, 1 \mathrm{H}, \mathrm{NH})$, 7.46 (s, 1H, 3-H), 7.85 (m, 2H, 6-H, 7-H), 8.36 (m, 2H, 5-H, 8-H), 12.88 (s, 1H, -OH), 13.39 (s, $1 \mathrm{H},-\mathrm{OH}) ;{ }^{13} \mathrm{C} \mathrm{NMR}\left(125 \mathrm{MHz}, \mathrm{CDCl}_{3}\right.$ ): $\delta=13.9$ (q, C-6'), 16.1 (q, $\mathrm{CH}_{2} \mathrm{CH}_{3}$ ), $18.5\left(\mathrm{t}, \mathrm{CH}_{2} \mathrm{CH}_{3}\right.$ ), 29.8 (t, C-2'), 37.6 (t, $\mathrm{CHCH}_{2}$ ), 46.2 (d, C-3'), 66.2 (d, C-5'), 69.0 (d, C-4'), 73.3 (d, $\mathrm{CHCH}_{2}$ ), 97.4 (d, C-1'), 111.9 (s, C-10a), 112.4 (s, C-4a), 126.1 (d, C-3), 127.0 (d, C-5), 127.1 (d, C-8), 133.5 (s, C-9a), 133.6 (s, C-8a), 134.2 (d, C-7), 134.5 (d, C-6), 144.2 (s, C-2), 152.2 (s, C-4), 157.6 (s, C-1), 186.5 (s, C-10), 187.3 (s, C-9); Anal. Calcd for $\mathrm{C}_{26} \mathrm{H}_{26} \mathrm{NO}_{8} \mathrm{~F}_{3}$ (537.5): C, 58.10; H, 4.88; N, 2.61. Found: C, 57.93; H, 4.92; N, 2.74\%.

\section{1,4-Dihydroxy-2-[1-O-(2,3,6-trideoxy-3-trifluoroacetamido-a-L-lyxo-hexopyranosyl)-butyl]-} 9,10-anthraquinone (polar) (29). In the same reaction (see 28) $22 \mathrm{mg}$ (25\%) of the polar $\alpha$ glycoside 29 was isolated, mp (softening) $128^{\circ} \mathrm{C}$. IR: $3420(\mathrm{OH}, \mathrm{NH}), 1717(\mathrm{C}=\mathrm{O}), 1623$ (quinone, chelated), $1588 \mathrm{~cm}^{-1}$ (aromat). ${ }^{1} \mathrm{H} \mathrm{NMR}(270 \mathrm{MHz}): \delta=0.98(\mathrm{t}, J=7.2 \mathrm{~Hz}, 3 \mathrm{H}$, $\left.\mathrm{CH}_{2} \mathrm{CH}_{3}\right), 1.31$ (d, $\left.J=6.5 \mathrm{~Hz}, 3 \mathrm{H}, 66^{\prime}-\mathrm{H}\right), 1.33-1.63\left(\mathrm{~m}, 2 \mathrm{H}, \mathrm{CH}_{2}\right), 1.75\left(\mathrm{t}, J=7.0 \mathrm{~Hz}, 2 \mathrm{H}, \mathrm{CH}_{2}\right)$, $1.78\left(\mathrm{dt}, J=13.4,3.5 \mathrm{~Hz}, 1 \mathrm{H}, 2^{\prime} \mathrm{a}-\mathrm{H}\right), 2.02$ (dd, $\left.J=13.4,5.2 \mathrm{~Hz}, 1 \mathrm{H}, 2^{\prime} \mathrm{e}-\mathrm{H}\right), 3.67$ (m, 1H, 3'-H), 
4.24 (q, $\left.J=6.5 \mathrm{~Hz}, 1 \mathrm{H}, 5^{\prime}-\mathrm{H}\right), 4.53\left(\mathrm{~m}, 1 \mathrm{H}, 3^{\prime}-\mathrm{H}\right), 4.75$ (d, $\left.J=3.5 \mathrm{~Hz}, 1 \mathrm{H}, 1^{\prime}-\mathrm{H}\right), 6.71$ (br d, $J=$ $8.0 \mathrm{~Hz}, 1 \mathrm{H}, \mathrm{NH}), 7.39$ (s, 1H, 3-H), 7.86 (m, 2H, 6-H, 7-H), 8.34 (m, 2H, 5-H, 8-H), 12.91 (s, $1 \mathrm{H}, \mathrm{OH}), 13.43(\mathrm{~s}, 1 \mathrm{H}, 2 \mathrm{OH})$. EIMS $\left(180^{\circ} \mathrm{C}\right): \mathrm{m} / z(\%)=537(3)[\mathrm{M}+], 442(2), 312(93), 269$ (100), 253 (19), 225 (12), 155 (16), 113 (11), 77 (6). HREIMS: $m / z=537.1614$ (Calcd. for $\left.\mathrm{C}_{26} \mathrm{H}_{26} \mathrm{~F}_{3} \mathrm{NO}_{8}, 537.1611\right)$.

2,3-Bis-[O-(2,3,6-trideoxy-3-trifluoroacetamido- $\alpha$-L-lyxo-hexopyranosyl)-methyl]-1,4-

dihydroxy-9,10-anthraquinone (31). The glycosylation of aglycone $\mathbf{3 0}^{55,56} 60 \mathrm{mg}(0.2 \mathrm{mmol})$ was performed as in the general procedure to afford $36 \mathrm{mg}(24 \%)$ of bis- $\alpha$-glycoside $31, \mathrm{mp} 130$ ${ }^{\circ} \mathrm{C}$ (softening). IR: $3420(\mathrm{OH}, \mathrm{NH}), 1719(\mathrm{C}=\mathrm{O}), 1623$ (quinone, chelated), $1589 \mathrm{~cm}^{-1}$ (arom.). ${ }^{1} \mathrm{H} \mathrm{NMR}\left(250 \mathrm{MHz}, \mathrm{CDCl}_{3}\right): \delta=1.18\left(\mathrm{~d}, J=6.5 \mathrm{~Hz}, 6 \mathrm{H}, 66^{\prime}-\mathrm{H}\right), 1.74-2.18\left(\mathrm{~m}, 1 \mathrm{H}, 2{ }^{\prime} \mathrm{a}-\mathrm{H}, 2^{\prime} \mathrm{e}-\mathrm{H}\right)$, $3.62\left(\mathrm{~m}, 2 \mathrm{H}, 3^{\prime}-\mathrm{H}\right), 4.23$ (q, $\left.J=6.5 \mathrm{~Hz}, 2 \mathrm{H}, 5^{\prime}-\mathrm{H}\right), 4.42\left(\mathrm{~m}, 2 \mathrm{H}, 3^{\prime}-\mathrm{H}\right), 4.78$ (d, J=11.2 Hz,1 H, $\left.\mathrm{CH}_{2}\right), 4.94\left(\mathrm{~d}, J=11.2 \mathrm{~Hz}, 1 \mathrm{H}, \mathrm{CH}_{2}\right), 5.15\left(\mathrm{~m}, J=4.0 \mathrm{~Hz}, 2 \mathrm{H}, 1^{\prime}-\mathrm{H}\right), 6.77$ (d, $J=8.4 \mathrm{~Hz} .2 \mathrm{H}$, $\mathrm{NH}), 7.84(\mathrm{~m}, 2 \mathrm{H}, 6-\mathrm{H}, 7-\mathrm{H}), 8.36(\mathrm{~m}, 2 \mathrm{H}, 5-\mathrm{H}, 8-\mathrm{H}), 13.52$ (s, 2H, $2 \mathrm{OH})$. Anal. Calcd for $\mathrm{C}_{32} \mathrm{H}_{32} \mathrm{~N}_{2} \mathrm{O}_{12} \mathrm{~F}_{6}$ (750.62): C, 51.21; H, 4.30; N, 3.73. Found: C, 50.97; H, 4.28; N, 3.75\%.

1,5-Dihydroxy-2,6-bis-[1-O-(2'-,3',6'-trideoxy-3'-trifluoroacetamido-a-L-lyxo-hexopyranosyl)-methyl]-9,10-anthraquinone (33) and the mixed $\alpha$-L-lyxo-hexopyranosyl- $\beta$-Llyxo-furanoside glycoside (34). Aglycone $\mathbf{3 2}^{54}$ (200 $\left.\mathrm{mg}, 0.67 \mathrm{mmol}\right)$ was glycosylated as described in the general procedure using $940 \mathrm{mg}(2.17 \mathrm{mmol})$ of sugar 2 and $780 \mathrm{mg}$ (3.04 mmol) of silver triflate to afford $84.1 \mathrm{mg}(24 \%)$ of less polar bis- $\alpha$-glycoside $33, \mathrm{mp} 258-260{ }^{\circ} \mathrm{C}$ and $30.8 \mathrm{mg}(9 \%)$ of the polar mixed glycoside, $34, \mathrm{mp} 238-240{ }^{\circ} \mathrm{C}$.

Data for 33. IR: 3430, $3290(\mathrm{OH}, \mathrm{NH}), 2978,2938(-\mathrm{CH}), 1710$ (CO), 1630 (quinone, chelated), $1425,1257,1170,980,800 \mathrm{~cm}^{-1} .{ }^{1} \mathrm{H} \mathrm{NMR}\left(250 \mathrm{MHz}, \mathrm{CDCl}_{3}\right): \delta=1.27(\mathrm{~d}, J=6.5 \mathrm{~Hz}, 6 \mathrm{H}, 2 \mathrm{x}$ 6'-H), 1.86 (dt, $\left.J=12.8,3.7 \mathrm{~Hz}, 2 \mathrm{H}, 2 \times 2^{\prime}-\mathrm{Ha}\right), 2.08$ (ddd, $J=12.8,6.0,1.0 \mathrm{~Hz}, 2 \mathrm{H}, 2$ x 2'-He), 3.64 (dd, $J=8.4,2.4 \mathrm{~Hz}, 2 \mathrm{H}, 2$ x 4'-H), 4.15 (q, $\left.J=6.5 \mathrm{~Hz}, 2 \times 5^{\prime}-\mathrm{H}\right), 4.51$ (dddd, $J=12.8,8.0$, 2.4, $\left.6 \mathrm{~Hz}, 2 \mathrm{H}, 2 \times 3^{\prime}-\mathrm{H}\right), 4.66\left(\mathrm{~d}, J=14.3 \mathrm{~Hz}, 2 \mathrm{H}, 2 \times \mathrm{CH}_{2}\right), 4.90\left(\mathrm{~d}, J=14.3 \mathrm{~Hz}, 2 \mathrm{H}, 2 \times \mathrm{CH}_{2}\right)$, $5.08(\mathrm{dd}, J=3.7,1.0 \mathrm{~Hz}, 2 \mathrm{H}, 2$ x l'-H), 6.71 (d, $J=8 \mathrm{~Hz}, 2 \mathrm{H}, 2$ x 4'-NH), 7.84 (d, $J=7.9 \mathrm{~Hz}, 2 \mathrm{H}$, 3-H, 7-H), 7.88 (d, $J=7.9 \mathrm{~Hz}, 2 \mathrm{H}, 4-\mathrm{H}, 8-\mathrm{H}), 13.04$ (s, 2H, 1-OH, 5-OH). ESIMS: 750 (37) $[\mathrm{M}+], 749(100)\left[\mathrm{M}^{+}-1\right]$; HREIMS: $m / z(\%)=773.1751$ (Calcd. for $\mathrm{C}_{32} \mathrm{H}_{32} \mathrm{~F}_{6} \mathrm{~N}_{2} \mathrm{O}_{12}+\mathrm{Na}$, 773.1752). Data for 34. IR: 3410, $3300(\mathrm{NH}, \mathrm{OH}), 2978,2920(-\mathrm{CH}), 1712(\mathrm{CO}), 1630$ (quinone, chelated), 1430, 1250, 1170, 980, $800 \mathrm{~cm}^{-1} .{ }^{1} \mathrm{H} \mathrm{NMR}\left(\mathrm{CDCl}_{3}, 500 \mathrm{MHz}\right): \delta=1.27(\mathrm{~d}, J=6.5 \mathrm{~Hz}$, 6H, 6'-H, 6"-H), 1.85 (dd, $\left.J=3.9,1 \mathrm{H}, 2^{\prime}-\mathrm{Ha}\right), 1.92$ (d, $\left.J=13.5 \mathrm{~Hz}, 1 \mathrm{H}, 2 "-\mathrm{Ha}\right), 2.08$ (ddd, $J=$ 1.0, 6.5, $12.8 \mathrm{~Hz}, 1 \mathrm{H}, 2^{\prime}-\mathrm{He}$ ), 2.28 (ddd, $J=4.3,13.5,7.8 \mathrm{~Hz}, 1 \mathrm{H}, 2$ "-He), 3.63 (br s, 1H, 4'-H), 3.83 (ddq, $J=5.1,5.9,6.5 \mathrm{~Hz}, 1 \mathrm{H}, 5 "-\mathrm{H}), 3.86$ (dd, $J=2.2,5.1 \mathrm{~Hz}, 1 \mathrm{H}, 4 "-\mathrm{H}), 4.13$ (q, $J=6.5$ Hz, 1H, 5'-H), 4.53 (m, 2H, 3'-H, 3"-H), 4.55 (d, J=12.2 Hz, 1H, CH $), 4.66$ (d, J=14.4 Hz, 1H, $\left.\mathrm{CH}_{2}\right), 4.90\left(\mathrm{~d}, J=14.4,1 \mathrm{H}, \mathrm{CH}_{2}\right), 5.00\left(\mathrm{~d}, J=12.2 \mathrm{~Hz}, 1 \mathrm{H}, \mathrm{CH}_{2}\right), 5.07\left(\mathrm{~d}, J=3.8 \mathrm{~Hz}, 1 \mathrm{H}, \mathrm{l}^{\prime}-\mathrm{H}\right)$, $5.44(\mathrm{~d}, J=4.0 \mathrm{~Hz}, 1 \mathrm{H}, 1 "-\mathrm{H}), 6.75(\mathrm{~d}, J=8.1 \mathrm{~Hz}, 1 \mathrm{H}), 7.40(\mathrm{~d}, J=8.1 \mathrm{~Hz}, 1 \mathrm{H}, \mathrm{NH}), 7.87$ (m, 4H, H-3, H-4, H-7, H-8), 12.96 (s, 1H, OH), 13.18 (s, 1H, OH); ${ }^{13} \mathrm{C}$ NMR (125 MHz, CDCl $): \delta$ $=16.6$ (q, C-6'), 19.4 (q, C-6"), 29.7 (t, C-2'), 38.9 (t, C-2"), 46.2 (d, C-3'), 50.9 (d, C-3"), 63.5 (d, C-5'), 64.0 (d, C-5"), 66.0 (d, $\left.\mathrm{CH}_{2}\right), 68.4$ (d, $\left.\mathrm{CH}_{2}\right), 89.8$ (d, C-4"), 96.7 (d, C-1'), 103.9 (d, C1"), 115.4 (s, C-1a, C-9a), 118.0 (s, C-4a, C-10a), 119.0 (d, C-4), 199.3 (d, C-8), 132.1 (s, C-2), 
132.8 (s, C-6), 134.6 (d, C-3), 135.0 (d, C-7), 160.3 (s, C-1), 160.8 (s, C-5), 187.9 (s, C-9), 188.0 $(\mathrm{s}, \mathrm{C}-10)$. EIMS $\left(180{ }^{\circ} \mathrm{C}\right): \mathrm{m} / z(\%)=750(0.5)\left[\mathrm{M}^{+}\right], 732(0.5), 706(0.5), 676(0.5), 621(1), 604$ (2), 593 (1), 508 (39), 300 (85), 284 (100), 266 (82), 226 (23), 113 (60). EIMS: 750 (42) [M ${ }^{+}$], 749 (100) $\left[\mathrm{M}^{+}-1\right]$; HREIMS: $m / z(\%)=773.1750$ (Calcd. for $\mathrm{C}_{32} \mathrm{H}_{32} \mathrm{~F}_{6} \mathrm{~N}_{2} \mathrm{O}_{12}+\mathrm{Na} 773.1752$ ).

\section{7-O-(2,3,6-Trideoxy-3-trifluoroacetamido- $\alpha$-L-lyxo-hexopyranosyl)-steffimycinone}

(36).

Steffimycinone $(4.1 \mathrm{mg}, 0.01 \mathrm{mmol})(35)$ was converted to the glycoside as described in the general procedure to afford $4.3 \mathrm{mg}(67 \%)$ of 36. ${ }^{1} \mathrm{H}$ NMR: $\delta=1.23\left(\mathrm{~d}, J_{5^{\prime}, 6^{\prime}}=6.6 \mathrm{~Hz}, 3 \mathrm{H}, 6^{\prime}-\mathrm{H}\right)$, $1.59\left(\mathrm{~s}, 3 \mathrm{H}, \mathrm{CH}_{3}\right), 1.91\left(\mathrm{dt}, J_{2^{\prime} \mathrm{a}, 2^{\prime} \mathrm{e}}=13.2, J_{2^{\prime} \mathrm{a}, 3^{\prime}}=12.4, J_{1^{\prime}, 2 \mathrm{a}^{\prime}}=3.7 \mathrm{~Hz}, 1 \mathrm{H}, 2^{\prime} \mathrm{a}-\mathrm{H}\right), 2.07\left(\mathrm{dd}, J_{2}{ }^{\prime} \mathrm{a}, 2^{\prime} \mathrm{e}\right.$ $\left.=13.2, J_{2}{ }^{\prime} \mathrm{e}, 3^{\prime}=5.0 \mathrm{~Hz}, 1 \mathrm{H}, 2^{\prime} \mathrm{e}-\mathrm{H}\right) .3 .55\left(\mathrm{~s}, 3 \mathrm{H}, \mathrm{OCH}_{3}\right), 3.64\left(\mathrm{~m}, 1 \mathrm{H}, 4{ }^{\prime}-\mathrm{H}\right), 3.71$ (m, $\left.1 \mathrm{H}, 8-\mathrm{H}\right)$, $3.98\left(\mathrm{~s}, 3 \mathrm{H}, \mathrm{OCH}_{3}\right), 4.24\left(\mathrm{q}, J_{5,6^{\prime}}=6.6 \mathrm{~Hz}, 1 \mathrm{H}, 5^{\prime}-\mathrm{H}\right), 4.44\left(\mathrm{~m}, 1 \mathrm{H}, 3^{\prime}-\mathrm{H}\right), 5.24\left(\mathrm{~m}, J_{\text {combined }}=9\right.$ $\mathrm{Hz}, 1 \mathrm{H}, 7-\mathrm{H}), 5.64\left(\mathrm{~d}, J_{1^{\prime}, 2^{\prime} \mathrm{a}}=3.7 \mathrm{~Hz}, 1 \mathrm{H}, 1^{\prime}-\mathrm{H}\right), 6.73$ (d, $\left.J_{1,3}=2.4 \mathrm{~Hz}, 1 \mathrm{H}, 3-\mathrm{H}\right), 6.77\left(\mathrm{~d}, J_{3^{\prime}, \mathrm{NH}}\right.$ $=8.5 \mathrm{~Hz}, 1 \mathrm{H}, \mathrm{NH}), 7.44\left(\mathrm{~d}, J_{1,3}=2.4 \mathrm{~Hz}, 1 \mathrm{H}, 1-\mathrm{H}\right), 8.35(\mathrm{~s}, 1 \mathrm{H}, 11-\mathrm{H}), 12.11(\mathrm{~s}, 1 \mathrm{H}, 4-\mathrm{OH})$, $12.91(\mathrm{~s}, 1 \mathrm{H}, 6-\mathrm{OH})$. EIMS $\left(180{ }^{\circ} \mathrm{C}\right): m / z(\%)=639$ (2) $[\mathrm{M}+], 477,(3), 451$ (9), 414 (16), 396 (30), 621 (1), 353 (63), 593 (1), 324 (25), 226 (100). HREIMS: $m / z(\%)=639.1564$ (Calcd. for $\left.\mathrm{C}_{29} \mathrm{H}_{28} \mathrm{~F}_{3} \mathrm{NO}_{12}, 639.1564\right)$.

\section{Acknowledgements}

We thank the German Science Foundation (DFG) for financial support and Prof. Federico-Maria Arcamone for helpful suggestions and gift of daunosamin hydrochloride.

\section{References}

1. Zhang, G.; Fang, L.; Zhu, L.; Zhong, Y.; Wang, P. G.; Sun, D. J. Med. Chem. 2006, 49, 1792 and references therein.

2. Kelly, T. R. Ann. Rep. Med. Chem. 1979, 14, 288.

3. Broadhurst, M. J.; Hassall, C. H.; Thomas, G. J. Chem. and Ind. 1985, 106.

4. Krohn, K. Angew. Chem. 1986, 98, 788-787; Angew. Chem. Int. Ed. 1986, 25, 790.

5. Stoodley, R. J. Second SCI/RSC Medical Chemistry Symposium; Spec. Publ. Royal Society of Chemistry, Cambridge, 1984, p134.

6. Thomson, R. H., Naturally Occurring Quinones III. Recent Advances, Chapman and Hall: London, 1987; pp 345-526.

7. Tamura, Y.; Kita, Y. J. Syn. Org. Chem. (Japan) 1988, 205.

8. Krohn, K.; Ekkundi, V. S., Total Synthesis of Enantiomerically Pure Anthracyclinones in Studies in Natural Product Chemistry, Stereoselective Synthesis Vol. 4 (Part C) Atta-urRahman, Ed., Elsevier: Amsterdam, 1989, pp 317-366. 
9. Krohn, K. Building Blocks for the Total Synthesis of Anthracyclinones In Progr. Chem. Org. Nat. Prod. Vol. 48, Herz, W.; Grisebach, H.; Kirby, G. W.; Tamm, Ch., Eds., Springer; Wien and New York, 1989; pp 37-88.

10. Kelly, T. R. Ed.; Tetrahedron Symposia in Print No. 17 1984, 40, 4537.

11. Lown, J. W. Chem. Rev. 1993, 165.

12. Krohn, K. Eur. J. Org. Chem. $2002,1351$.

13. Cambie, R. C.; Rutledge, P. S.; Woodgate, P. D. Austral. J. Chem. 1992, 45, 483.

14. Brockmann, H. Fortsch. Chem. Org. Naturst. 1963, 21, 121.

15. El Khadem, H. S., Anthracycline Antibiotics, Academic Press: New York, 1982.

16. Abdella, B. R. J.; Fisher, J. Environ. Health Perspect. 1985, 64, 3.

17. Arcamone, F. Doxorubicin, Academic Press: New York, 1981.

18. Anthracyclines. Current Status and New Developments; Crooke, S. T.; Reich, S. D., Eds.: Academic Press: New York, 1980.

19. Gesson, J.-P.; Mondon, M. Actual. Chim. Thér. 1986, 13, 161.

20. Arcamone, F. Cancer Res. 1985, 45, 5995.

21. Arcamone, F. The Development of New Antitumor Anthracyclines in Anticancer Agents based on Natural Products Models, Cassidy, J. M.; Dowes, J. D. Eds, vol. 16, Academic Press: New York, 1980, pp 1-41.

22. Strauss, D. G. Pharmazie 1987, 42, 289.

23. Priebe, W. Ed; Anthracycline Antibiotics, New Analogues, Methods of Delivery, and Mechanisms of Action, in ACS Symposium Series, Vol. 574, 1995.

24. Suarato, A.; Angelucci, F.; Bargiotti, A. Chim. Oggi 1990, 8, 9.

25. Ikeda, D.; Ajito, K. Yuki Gosei Kagaku Kyokaishi 1991, 49, 1002.

26. Takagi, Y.; Tsuchiya, T.; Miyake, T.; Takeuchi, T.; Umezawa, S. Yuki Gosei Kagaku Kyokaishi 1992, 50, 131.

27. Monneret, C. Ann. Pharm. Fr. 1997, 55, 5.

28. Taatjes, D. J.; Fenick, D. J.; Gaudiano, G.; Koch, T. H. Curr. Pharm. Des. 1998, 4, 203.

29. Vogel, P. Curr. Org. Chem. 1998, 2, 255.

30. Arcamone, F.; Cassinelli, G. Curr. Med. Chem. 1998, 5, 391.

31. Suarato, A.; Angelucci, F.; Geroni, C. Curr. Pharm. Des. 1999, 5, 217.

32. Michel, S.; Desbene, S.; Gesson, J.-P.; Monneret, C.; Tillequin, F. Stud. Nat. Prod. Chem. 2000, 21, 157.

33. Monneret, C. Eur. J. Med. Chem. 2001, 36, 483.

34. Binaschi, M.; Bigioni, M.; Cipollone, A.; Rossi, C.; Goso, C.; Maggi, C. A.; Capranico, G.; Animati, F. Curr. Med. Chem.: Anti-Cancer Agents 2001, 1, 113.

35. Le Sann, C. Nat. Prod. Rep. 2006, 23, 357.

36. (a) Arcamone, F. C., Anthracyclines in Anticancer Agents from Natural Products; Gragg, G. M.; Kingston, D. G. I.; Newman, D. J., Eds.; Taylor \& Francis, Boca Raton, 2005, pp 299320. (b) Anthracyclines, Chemistry and Biology, in Topics Curr. Chem.: Krohn, K., Ed.; Springer: Heidelberg, 2008; Vol. 1. 
37. Kita, Y.; Kirihara, M.; Sekihachi, J.; Okunaka, R.; Sasho, M.; Mohri, S.; Honda, T.; Akai, S.; Tamura, Y.; Shimooka, K. Chem. Pharm. Bull. 1990, 38, 1836.

38. Menyhart, M; Koever, K.; Sztaricskai, F. J. Carbohydr. Chem. 1990, 9, 253.

39. Olsufyeva, E. N.; Backinowsky, L. V. Tetrahedron Lett. 1990, 31, 4805.

40. Kita, Y.; Kirihara, M.; Fujii, Y.; Okunaka, R.; Akai, S.; Maeda, H.; Tamura, Y.; Shimooka, K.; Ohishi, H.; Ishida, T. Chem. Pharm. Bull. 1991, 39, 857.

41. Tolstikov, G. A.; Shul'ts, E. E.; Vafina, G. F.; Spirikhin, L. V. Zh. Org. Khim. 1992, $28,192$.

42. Acosta, J. C.; Caballero, E.; Gravalos, D. G.; Medarde, M.; Sahagun, H.; Stoodley, R. J.; Tome, F. Bioorg. Med. Chem. Lett. 1997, 7, 2955.

43. Cameron, D. W.; Feutrill, G. I.; Griffiths, P. G. Aust. J. Chem. 2000, 53, 25.

44. Rho, Y. S.; Kim, W.-J.; Yoo, D. J.; Kang, H. S.; Chung, S.-R. Bull. Korean Chem. Soc. 2001, 22, 963.

45. Priebe, W.; Grynkiewicz, G.; Neamati, N. Monatsh. Chem. 1991, 122, 419.

46. Cameron, D. W.; Griffiths, P. G. Tetrahedron Lett. 1993, 34, 4685.

47. Toshima, K.; Hasegawa, M.; Shimizu, J.; Matsumura, S. ARKIVOC 2004, xiii, 28.

48. Horton, D.; Weckerle, W. Carbohydr. Res. 1975, 44, 227.

49. Hanessian, S.; Banoub, J. Carbohydr. Res. 1975, 44, C14.

50. (a) Arcarnone, F.; Bargiotti, A.; Cassinelli, G.; Redaelli, S.; Hanessian, S.; Di Marco, A.; Casazza, A. B.; Dasdia, T.; Necco, A.; Reggiani, P.; Supino, R. J. Med. Chem. 1976, 19, 733. (b) Broadhurst, M. J.; Hassall, C. H.; Thomas, G. J. Chem. Commun. 1982, 158. (c) Di Marco, A.; Casazza, A. M.; Giuliani, F.; Pratesi, G.; Arcamone, F.; Bernardi, L.; Franchi, G.; Giardino, P.; Patelli, B.; Penco, S. Cancer Treat. Rep. 1978, 62, 375.

51. Arcamone, F.; Bernardi, L.; Patelli, B.; Giardino, P.; Di Marco, A.; Casazza, A. M.; Soranzo, C.; Pratesi, G. Experientia 1978, 34, 1255.

52. Arcamone, F. Lloydia 1977, 40, 45.

53. Bredereck, K.; Banzhaf, L.; Koch, E. Chem. Ber. 1972, 105, 1062.

54. Bredereck, K.; Metwally, S. A.; Koch, E.; Weckmann, R. Liebigs Ann. Chem. 1975, 972.

55. Krohn, K.; Hemme, C. Liebigs Ann. Chem. 1979, 19.

56. Krohn, K.; Hemme, C. Liebigs Ann. Chem. 1979, 35.

57. El Khadem, H.; Matsuura, D. Carbohydr. Res. 1982, 99, 143.

58. Kelly, R. C.; Schletter, I.; Koert, J. M.; MacKellar, F. A.; Wiley, P. F. J. Org. Chem. 1977, $42,3591$.

59. Krohn, K.; Behnke, B. Chem. Ber. 1980, 113, 2994.

60. Krohn, K.; Klimars, M.; Köhle, H. J.; Ebeling, E. Tetrahedron 1984, 40, 3677.

61. Krohn, K.; Müller, U.; Priyono, W.; Sarstedt, B.; Stoffregen, A. Liebigs Ann. Chem. 1984, 306.

62. Jin, G.-Z.; Song, G.-Y.; Zheng, X.-G.; Kim, Y.; Sok, D.-E.; Ahn, B.-Z. Arch. Pharm. Res. 1998, 21, 198; Chem. Abstr. 1998, 129, 36203. 